This item was submitted to Loughborough's Research Repository by the author.

Items in Figshare are protected by copyright, with all rights reserved, unless otherwise indicated.

\title{
An unstructured-mesh atmospheric model for nonhydrostatic dynamics: towards optimal mesh resolution
}

PLEASE CITE THE PUBLISHED VERSION

http://dx.doi.org/10.1016/j.jcp.2015.03.054

\section{PUBLISHER}

(c) Elsevier Inc.

\section{VERSION}

AM (Accepted Manuscript)

\section{PUBLISHER STATEMENT}

This work is made available according to the conditions of the Creative Commons Attribution-NonCommercialNoDerivatives 4.0 International (CC BY-NC-ND 4.0) licence. Full details of this licence are available at: https://creativecommons.org/licenses/by-nc-nd/4.0/

\section{LICENCE}

CC BY-NC-ND 4.0

\section{REPOSITORY RECORD}

Szmelter, Joanna, Zhao Zhang, and Piotr K. Smolarkiewicz. 2015. "An Unstructured-mesh Atmospheric Model for Nonhydrostatic Dynamics: Towards Optimal Mesh Resolution”. Loughborough University. https://hdl.handle.net/2134/18284. 


\title{
An unstructured-mesh atmospheric model for nonhydrostatic dynamics: towards optimal mesh resolution
}

\author{
Joanna Szmelter ${ }^{a}$, Zhao Zhang ${ }^{b}$, and Piotr K. Smolarkiewicz ${ }^{c}$

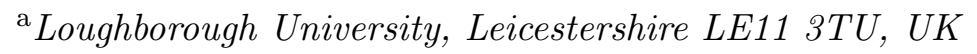 \\ ${ }^{\mathrm{b}}$ Heriot-Watt University, Edinburgh, EH14 4AS, UK \\ ${ }^{\mathrm{c}}$ European Centre For Medium Range Weather Forecasts, Reading, RG2 9AX, UK
}

\begin{abstract}
The paper advances the limited-area anelastic model [Smolarkiewicz et al., J. Comput. Phys., 254 (2013) 184] for investigation of nonhydrostatic dynamics in mesoscale atmospheric flows. New developments include the extension to a tetrahedral-based median-dual option for unstructured meshes and a static mesh adaptivity technique using an error indicator based on inherent properties of the Multidimensional Positive Definite Advection Transport Algorithm (MPDATA). The model employs semi-implicit nonoscillatory forward-in-time integrators for soundproof PDEs, built on MPDATA and a robust non-symmetric Krylov-subspace elliptic solver. Finitevolume spatial discretisation adopts an edge-based data structure. Simulations of stratified orographic flows and the associated gravity-wave phenomena in media with uniform and variable dispersive properties, verify the advancement and demonstrate the potential of heterogeneous anisotropic discretisation with large variation in spatial resolution for study of complex stratified flows that can be computationally unattainable with regular grids.
\end{abstract}

Key words: unstructured mesh atmospheric models, nonoscillatory forward-in-time schemes, anelastic equations, orographic flows, critical layer PACS:

\footnotetext{
* Corresponding Author.

Email address: j.szmelter@lboro.ac.uk (Joanna Szmelter).
} 


\section{Introduction}

Historically, atmospheric models across scales and foci of interests were dominated by finite-difference and spectral-transform methods for spatial discretisation of their governing PDEs. In particular, finite-difference methods operating on regular rectangular grids have prevailed in small and mesoscale models for research of cloud processes and flows over complex terrain [36], with terrain fitted grids mimicked by continuous mappings [13] and horizontal resolution refinement delegated to nested grids [6]. These techniques are still standard in computational studies from planetary boundary layer to regional climate $[48,12]$.

Pioneered by the OMEGA model [1] for forecasting high-impact weather, air quality, and environmental hazard, there has been a growing interest in modelling atmospheric flows on unstructured meshes and utilising flexible mesh adaptivity. Interest in unstructured meshing per se dates back to the nineteen sixties [58], in the context of homogeneous discretisation for global flows. However, a broader research of flexible mesh adaptivity is relatively new — cf. the collection of papers [29] and seminars [17] — and adaptive-mesh atmospheric models have not yet achieved the maturity and acceptance of structured-grid models, commonly used in research and operations. ${ }^{1}$ The meteorological community appears to be concerned with possible drawbacks of anisotropic heterogeneous discretisation for fundamental balances and wave propagation underlaying atmospheric dynamics; see [10] for a substantive discussion. This poses new challenges to flexible mesh adaptivity, largely developed in the engineering community for neutrally stratified non-rotating flows [59].

However, unstructured-meshes and mesh adaptivity offer flexibility unmatched by the established techniques operating on regular grids. Even though the latter enable computationally efficient static and dynamic mesh adaptivity via continuous mappings [31,56,21,3,4], their rigid connectivity imposes stringent constraints on adapted grids. Flexible unstructured meshes relax the constraints and offer alternative means for optimising variable resolution required for improved representation of complex physical processes in atmospheric flows. Generally, such flows evince the multiplicity of scales ranging from a fraction of a millimetre where dissipation occurs, to tens of thousands of kilometres where planetary weather and climate take place. For some atmospheric processes, that are still insufficiently understood in spite of their relevance to weather conditions in populated areas, flexible mesh adaptivity can improve solution realizability, and thus cognition and predictability, at-

\footnotetext{
1 According to the Thomson Reuters Web of Science, among nearly 900 papers published since 1985 on the topic of "mesh adaptivity" less than $1.5 \%$ falls into the category "meteorology atmospheric sciences".
} 
tainable with available computational resources. Examples include weather in long winding valleys and mountainous areas, onset and evolution of radiation fog or stratiform clouds, precipitation or extreme events forecasting. This paper advances the three-dimensional, nonhydrostatic, limited-area model designed for flexible fully unstructured meshes [45] by introducing its implementation on tetrahedral based median-dual meshes that enable a wide range of mesh refinement strategies.

The current work extends our earlier developments aiming at generalisation of the non-oscillatory forward-in-time (NFT) integrators to unstructured meshes. The NFT integrators were first proposed for finite-difference atmospheric models [39] and were proven for a range of computational studies from groundwater flows [19], through all scale atmospheric dynamics [46], to magnetic cycles in global stellar convection [14]. The roots of the NFT schemes for unstructured meshes are in the derivation from first principles of the finitevolume multidimensional positive definite advection transport algorithm (MPDATA) together with the stability and convexity theory, convergence analysis, and several MPDATA options [41,42]. ${ }^{2}$ Further developments included an unstructured-mesh finite-volume NFT framework for gas dynamics with a selection of techniques for mesh adaptivity [50,43] originally developed for engineering flows $[59,49,51]$. The applicability of NFT methods to gas dynamics was demonstrated through verifications against benchmarks for all-speed aerodynamic flows with favourable comparisons to established solutions (theoretical and numerical) and convergence studies. Subsequent developments of the unstructured meshes based NFT integrators focused on meteorological applications producing models for simulating idealised hydrostatic dynamics of the planetary atmosphere [52], reduced 2D soundproof models for simulation of nonhydrostatic gravity-wave dynamics $[53,44]$ and their consequent generalisation to 3D mesoscale modelling of nonhydrostatic dynamics [45] — all supported with extensive verification studies.

The underlying concept of the numerical model used in the current study is presented in detail in [45]. In the model, all dependent variables are colocated, benefiting memory and communication requirements compared to staggered arrangements. This also facilitates implicit representation of buoyant modes. Generally, NFT labels a class of second-order-accurate two-timelevel schemes (of the Cauchy-Kowalewski type, cf. section 19 in [54]) for integrating fluid PDEs that are built on nonlinear advection schemes such as MPDATA. The MPDATA schemes control numerical oscillations in the sense of high-resolution methods [33], and this assures the nonlinear stability for

$\overline{2}$ The potential of MPDATA for unstructured mesh modelling was recognised much earlier: first in context of mantle convection models [5], and then in the area of weather and environmental modelling [1]; however the derivation in [42] is the first to encompass error compensating terms required for unstructured meshes. 
the co-located arrangement of dependent variables (cf. appendix A1 in [42]). Furthermore, in the NFT solver, MPDATA provides implicit turbulence modelling capability to the full set of equations. The implicit large eddy simulation (ILES) properties of MPDATA-based high-Reynolds-number solvers were widely documented in the context of structured grids [24,25,7,26,55]. More recently, they were also demonstrated for unstructured meshes [53,44,45] and verified against published data and LES/ILES results generated with established codes in [45]. For unstructured meshes with large variation in spatial resolution, the ILES capability is especially practical, as it circumvents evaluation of explicit subgrid-scale models and construction of commutative filters for LES [27], in particular, and does not impede the stability of calculations.

The preceding works on the nonhydrostatic unstructured-mesh NFT models $[53,44,45]$ verified the excellent accuracy of the finite-volume approach, using benchmarks from both analytic and laboratory results, and comparing unstructured-mesh results to the corresponding results obtained with the structured-grid EULAG model [32]. The previously studied physical problems representative of mesoscale dynamics included nonhydrostatic mountain waves at weak and strong background stratification (with linear and nonlinear flow responses, respectively), amplification and breaking of deep stratospheric gravity waves, low Froude number flows past steep isolated 3D mountain, and evolution of convective planetary boundary layer. Here, we advance simulation of flows past complex terrain and associated gravity-wave phenomena. In particular, we consider the special problem of a stratified flow past a twoscale slab-symmetric mountain - known to bifurcate into a qualitatively incorrect solution at coarse resolutions - to demonstrate the capabilities of unstructured meshes and static adaptive mesh refinement. Notably, an a posteriori error indicator that drives the mesh refinement arises from the inherent properties of MPDATA [50]. We also revisit 3D low Froude number flows to verify the accuracy of tetrahedral discretisation. Finally, we introduce to the unstructured-mesh NFT modelling the problem of gravity-wave critical-level interaction. This problem is essentially stiff, as the vertical wavelength of a vertically propagating wave diminishes to zero upon approaching the critical level, so processes acting on small scales become important and even an infinitesimal amplitude wave becomes nonlinear. The problem exposes benefits of unstructured meshes particularly well as it calls for a high degree of refinement in a small portion of the computational domain.

The remainder of the paper is organised as follows. The numerical methodology is outlined in the next section. Section 3 presents the procedure utilised for static adaptivity applied to the so-called Schär [34] problem for two-scale mountain wave. Section 4 examines the accuracy of tetrahedral meshing in the context of a strongly stratified flow past a steep isolated hill. Section 5 quantifies the model accuracy for simulations with highly anisotropic and inhomogeneous meshes in the context of critical-level phenomena. Section 6 
concludes the paper.

\section{Nonoscillatory forward in time integration}

\subsection{Governing equations}

The numerical solutions presented in the paper are obtained by integrating either the anelastic Lipps-Hemler PDEs $[22,23]$ or their incompressible Boussinesq limit [47], in absence of viscosity, friction, diabatic effects and planetary rotation. In both cases, the governing conservation laws of mass, momentum and thermodynamic properties can be compactly written as

$$
\begin{aligned}
& \nabla \cdot\left(\mathbf{u} \rho_{o}\right)=0, \\
& \frac{\partial \rho_{o} u^{I}}{\partial t}+\nabla \cdot\left(\mathbf{u} \rho_{o} u^{I}\right)=\rho_{o}\left(-\frac{\partial \pi^{\prime}}{\partial x^{I}}+g \frac{\Theta^{\prime}}{\Theta_{o}} \delta_{I 3}\right), \\
& \frac{\partial \rho_{o} \Theta^{\prime}}{\partial t}+\nabla \cdot\left(\mathbf{u} \rho_{o} \Theta^{\prime}\right)=-\rho_{o} \mathbf{u} \cdot \nabla \Theta_{e} .
\end{aligned}
$$

Here, $\rho, \Theta$ and $\pi$ are, respectively, the density, potential temperature and normalised pressure, $\pi=p / \rho_{o}$ with $p$ marking the pressure. For $I=1,2$, the velocity components refer to the horizontal directions $x^{1} \equiv x, x^{2} \equiv y$ with $u^{1} \equiv$ $u, u^{2} \equiv v$; whereas in the vertical $I=3$ with $x^{3} \equiv z$ and $u^{3} \equiv w$. Subscripts o denote the static reference state, while primes denote perturbations with respect to a hydrostatically balanced ambient state distinguished with the subscript $e$. In the anelastic case vertical profiles $\rho_{o}(z)$ and $\Theta_{o}(z)$ are defined in terms of a constant stratification $S_{o} \equiv d \ln \Theta_{o} / d z=N^{2} / g$, where $g(=$ $9.81 \mathrm{~ms}^{-2}$ ) and $N$ denote the gravitational acceleration and the buoyancy frequency, respectively. In the Boussinesq limit, $\rho_{o}$ and $\Theta_{o}$ are constant. In examples discussed in this paper the ambient states assume either constant or linearly decreasing with height wind $U(z)$ and thermodynamic profiles defined by $S_{o}$ according to the hydrostatic balance of the governing PDEs.

\subsection{Semi-implicit integrator}

For the construction of numerical solution to the momentum and the potential temperature equations in (1), the generic NFT template algorithm is applied

$$
\begin{aligned}
\forall_{i, n} \quad \psi_{i}^{n+1} & =\mathcal{A}_{i}\left(\psi^{n}+0.5 \delta t R^{n}, \mathcal{V}^{n+1 / 2}, \rho_{o}\right)+0.5 \delta t R_{i}^{n+1} \\
& \equiv \mathcal{A}_{i}\left(\widetilde{\psi}, \boldsymbol{V}^{n+1 / 2}, \rho_{o}\right)+0.5 \delta t R_{i}^{n+1}
\end{aligned}
$$




$$
\equiv \widehat{\psi}_{i}+0.5 \delta t R_{i}^{n+1}
$$

with $\psi$ symbolising a dependent flow variable $u^{I}$ or $\Theta^{\prime}$ and $R$ denoting the ratio of the associated right-hand-side (rhs) in (1) to $\rho_{o}$. Indices $n$ and $i$ have their usual meaning of the temporal and spatial positions. The operator $\mathcal{A}$ is a shorthand for the edge-based MPDATA [42], and it already accounts for a division of conservative variables $\rho_{o} \psi$ by $\rho_{o}$. The $\boldsymbol{V}^{n+1 / 2}$ argument of $\mathcal{A}$ denotes an $\mathcal{O}\left(\delta t^{2}\right)$ estimate of $\rho_{o} \mathbf{u}$ at $t+0.5 \delta t$; whereas $R^{n+1}$ is a second-order-accurate finite volume representation of $R$; see [46] for extended discussion.

In the default form, the solution commences with the linear extrapolation of the advective mass flux

$$
\boldsymbol{V}_{i}^{n+1 / 2}=1.5 \boldsymbol{V}_{i}^{n}-0.5 \boldsymbol{V}_{i}^{n-1}
$$

followed by the computation of auxiliary variables

$$
\begin{aligned}
& \widetilde{\mathbf{u}}_{i}=\mathbf{u}_{i}^{n}-0.5 \delta t\left(\nabla \pi^{\prime}-g \frac{\Theta^{\prime}}{\Theta_{o}} \mathbf{k}\right)_{i}^{n}, \\
& \widetilde{\Theta}_{i}^{\prime}=\Theta_{i}^{\prime n}-0.5 \delta t\left(\mathbf{u} \cdot \nabla \Theta_{e}\right)_{i}^{n},
\end{aligned}
$$

and their subsequent MPDATA transport supplying $\widehat{\mathbf{u}}$ and $\widehat{\Theta^{\prime}}$ for $(2)$; here $\mathbf{k}=\nabla z$ denotes the unit vector in the vertical direction. In consequence, the realisations of (2) for the momentum and potential temperature are

$$
\begin{aligned}
& \mathbf{u}_{i}^{n+1}=\widehat{\mathbf{u}}_{i}-0.5 \delta t\left(\nabla \pi^{\prime}-g \frac{\Theta^{\prime}}{\Theta_{o}} \mathbf{k}\right)_{i}^{n+1}, \\
& \Theta_{i}^{\prime n+1}=\widehat{\Theta}_{i}^{\prime}-0.5 \delta t\left(\mathbf{u} \cdot \nabla \Theta_{e}\right)_{i}^{n+1}
\end{aligned}
$$

The resulting system (5)-(6) is implicit, because $\nabla \pi^{\prime n+1}, \Theta^{\prime n+1}$ and components of $\mathbf{u}^{n+1}$ are unknown on the rhs. Due to the co-location of dependent variables and linearity of the rhs, the implicitness of (5) with respect to $\Theta^{\prime n+1}$ can be readily removed. Inserting (6) in (5), and combining all terms dependent on $\mathbf{u}^{n+1}$ on the left-hand-side (lhs) produces

$$
\mathbf{L} \mathbf{u}_{i}^{n+1}=\widehat{\mathbf{u}}_{i}-\left.0.5 \delta t \nabla \pi^{\prime}\right|_{i} ^{n+1}
$$

where $\mathbf{L}$ symbolises a $3 \times 3$ matrix of known coefficients. Consequently, (7) can be written in a closed form as

$$
\mathbf{u}_{i}^{n+1}=\widehat{\widehat{\mathbf{u}}}_{i}-0.5 \delta t\left(\mathbf{C} \nabla \pi^{\prime}\right)_{i}^{n+1},
$$


where $\widehat{\hat{\mathbf{u}}}=\mathbf{L}^{-1} \widehat{\mathbf{u}}$ symbolises the modified explicit part of the solution, and $\mathbf{C} \equiv \mathbf{L}^{-1}$. For the applications addressed in the formulae in section 3.2 of [45] hold, upon which the components of $\widehat{\mathbf{u}}$ are:

$$
\hat{\hat{u}}=\hat{u}, \quad \hat{\hat{v}}=\hat{v}, \quad \hat{\hat{w}}=\left(\hat{w}+0.5 \delta t \beta \hat{\Theta}^{\prime}\right)\left[1+0.25 \delta t^{2} \beta \partial_{z} \Theta_{e}\right]^{-1},
$$

with $\beta \equiv g / \Theta_{o}$; whereas the only nonzero entries of $\mathbf{C}$ are

$$
C^{x x}=C^{y y}=1, \quad C^{z z}=\left[1+0.25 \delta t^{2} \beta \partial_{z} \Theta_{e}\right]^{-1} .
$$

In order to complete the solution for $\mathbf{u}$ in (8), the discrete elliptic Poisson problem for $\pi^{\prime}$ is formed by applying the discrete divergence operator to $\rho_{o} \times(8)$ and noting that $\nabla_{i} \cdot\left(\rho_{o} \mathbf{u}\right)^{n+1}$ must vanish on the lhs due to the continuity constraint in (1), thus resulting in

$$
\forall_{i} \quad \nabla_{i} \cdot \rho_{o}\left(\widehat{\mathbf{u}}-0.5 \delta t \nabla \pi^{\prime n+1}\right)=0 .
$$

The Poisson problem (11) is solved (subject to suitable boundary conditions) to a specified tolerance using the generalised conjugate residual algorithm, as described in [44]. The solution to (11) completes (8), upon which the potential temperature is updated according to (6). A simpler NFT integrator with explicit representation of the buoyant modes is available as an option (section 3.3 in [45]); however, the outlined semi-implicit scheme is more accurate [8,57] and, thus, exclusively used in this study. The summarised formulation of the semi-implicit integrator, is a special case of a general procedure admitting various PDE systems with multiplicity of the forces on the rhs; cf. $[32,46]$ and references therein.

\subsection{Spatial discretisation}

The spatial discretisation assumed in the template algorithm (2) uses the edge-based median-dual finite volume approach [42] integrating the generic physical form of the governing PDE over arbitrarily-shaped cells, with all discrete differential operators evaluated from the Gauss divergence theorem; cf. section 3.4 in [45]. A schematic of the edge-based data structure for an arbitrary hybrid mesh on a 2D plane is shown in Figure 1. The median-dual finite volume approach constructs the control volume containing the node $i$ by joining the barycentres of polygonal mesh cells encompassing the node $i$ with the midpoints of the edges originating in the node $i$. Having defined the mesh in planar geometry, all geometric elements such as cell volume, cell face area, and normals are evaluated from vector calculus. All dependent variables are co-located in the nodes. 


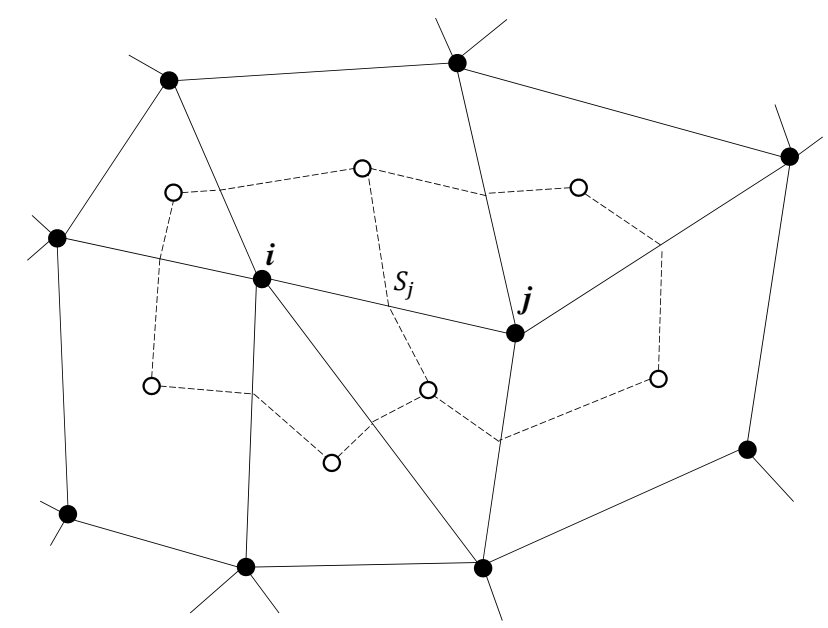

Fig. 1. The edge-based, median-dual approach in 2D. The edge connecting nodes $i$ and $j$ of the primary polygonal mesh pierces, precisely in its middle, the face $S_{j}$ shared by computational dual cells surrounding nodes $i$ and $j$; open circles represent barycentres of the primary mesh, while solid and dashed lines mark primary and dual meshes, respectively.



Fig. 2. The edge-based median-dual approach in 3D. The edge connecting nodes $i$ and $j$ of the primary mesh pierces (at the edge centre) the face $S_{j}$ of a computational (dual) cell surrounding node $i$; open circles represent barycentres of polyhedral cells surrounding the edge. Dashed lines mark a fragment of the dual mesh.

The construction of a dual mesh in three dimensions is similar, yet providing an illustration equivalent to Fig. 1 is hardly possible. Instead, Fig. 2 shows the edge $i \rightarrow j$ connecting nodes $i$ and $j$, intersected exactly in the middle by the face $S_{j}$ of a dual cell containing node $i$. The construction of the face $S_{j}$ involves first the identification of the polyhedral elements belonging to 
the primary mesh that share the edge $i \rightarrow j$. Next, the polyhedral barycentres and the centres of polygonal faces, through which neighbouring polyhedra are jointed, are connected with each other and with the mid-point of the edge $i \rightarrow j$, as indicated by dashed lines in Fig. 2, to form an umbrella like face of the $3 \mathrm{D}$ dual mesh.

\section{MPDATA driven mesh adaptivity}

The finite volume discretisation with the edge-based data structure is well suited for investigating mesh refinement. In particular, this includes a flexible use of mesh adaptivity techniques, for which an explicit analytic form of the leading truncation error arising from MPDATA can be exploited. For the reader's convenience, the next subsection summarises the finite-volume MPDATA, whereas the MPDATA based error indicators for mesh refinement are discussed in subsection 3.2 and subsequently illustrated in subsection 3.3.

\subsection{Highlights of finite-volume MPDATA}

In essence, MPDATA is an iterative application of the first-order accurate upwind scheme, where the first iteration uses physical velocities while all subsequent iterations compensate the leading truncation error of the preceding iteration using analytically derived pseudo-velocities and most recent iterates of the transported field. The MPDATA approach encompasses a number of schemes, however, even the simplest (basic) algorithm with one corrective iteration is conservative, sign-preserving and at least second-order accurate.

The explicit part of the solution in (2) applies MPDATA operator to an auxiliary variable $\widetilde{\psi}$. Integrating $\widetilde{\psi}$ over the volume of a cell surrounding node $i$ results in

$$
\tilde{\psi}_{i}^{(k)}=\tilde{\psi}_{i}^{(k-1)}-\frac{\delta t}{\vartheta_{i}} \sum_{j=1}^{l(i)} F_{j}^{\perp}{ }^{(k)}\left(\widetilde{\psi}_{i}^{(k-1)}, \widetilde{\psi}_{j}^{(k-1)}, \mathcal{V}_{j}^{\perp}{ }^{(k)}\right) S_{j} .
$$

Here the summation is over edges connecting node $i$ with one of its immediate neighbours $j$. There are $l(i)$ edges connecting the node $i$ with its neighbours, and $S_{j}$ refers both to the cell face pierced by the $j$ th edge and its surface area. $F_{j}^{\perp}$ is the mean normal flux through the cell face $S_{j}$ averaged over temporal increment $\delta t$ and $\mathcal{V}_{j}^{\perp}$ is the corresponding normal advective mass flux evaluated at the cell face. The cell volume $\vartheta_{i}$ contains node $i$. Furthermore, $k=1, . ., \mathcal{K}$

numbers the MPDATA iterations such that 


$$
\begin{aligned}
& \widetilde{\psi}^{(0)} \equiv \widetilde{\psi}^{n}, \quad \widetilde{\psi}^{(\mathcal{K})} \equiv \widetilde{\psi}^{n+1}, \\
& \mathcal{V}^{\perp}(k+1)=\mathcal{V}^{\perp}\left(\boldsymbol{\mathcal { V }}^{(k)}, \widetilde{\psi}^{(k)}, \nabla \widetilde{\psi}^{(k)}\right) ;\left.\quad \mathcal{V}^{\perp(1)} \equiv \mathcal{V}^{\perp}\right|^{n+1 / 2}
\end{aligned}
$$

All normal fluxes $F_{j}^{\perp^{(k)}}$ assume the functional form of the generic upwind flux

$$
F_{j}^{\perp}{ }^{(k)}\left(\widetilde{\psi}_{i}^{(k-1)}, \widetilde{\psi}_{j}^{(k-1)}, \mathcal{V}_{j}^{\perp(k)}\right)=\left[\mathcal{V}_{j}^{\perp(k)}\right]^{+} \widetilde{\psi}_{i}^{(k-1)}+\left[\mathcal{V}_{j}^{\perp^{(k-1)}}\right]^{-} \widetilde{\psi}_{j}^{(k-1)}
$$

where the nonnegative/nonpositive parts

$$
\left[\mathcal{V}_{j}^{\perp}{ }^{(k)}\right]^{+} \equiv \max \left[0, \mathcal{V}_{j}^{\perp^{(k)}}\right], \quad\left[\mathcal{V}_{j}^{\perp^{(k-1)}}\right]^{-} \equiv \min \left[0, \mathcal{V}_{j}^{{ }^{(k-1)}}\right]
$$

always coincide with outflow/inflow from the $i$ th cell.

MPDATA relies on expressions specifying the functional dependence (14) of the $\mathcal{V}^{\perp(k+1)}$ iterates of the normal advective mass flux. Generally, their explicit forms derive from the modified equation for the upwind advection, generated by expanding advectors $\mathcal{V}$ and advectees $\psi$ entering the scheme about a common point $(\mathbf{x}, t)$ and applying the Cauchy-Kowalewski procedure [54] to express higher-order temporal partial derivatives by the spatial partial derivatives $[39,40]$. This reveals the explicit form of the leading truncation-error flux of a predominantly diffusive character. Noting that the form of the error is an attribute of the functional form of upwind differencing - i.e., it remains the same for all $k$ in (12) — and writing the error flux as the advective flux of $\widetilde{\psi}$, defines the error-compensating pseudo-velocity in (14) as $\mathcal{V}^{{ }^{(k+1)}} \equiv-(\text { Error } / \widetilde{\psi})^{(k)}$. In particular, for the median dual meshes used here, the expression for the pseudo-velocity derived in [42] reduces to

$$
\begin{aligned}
\left.\mathcal{V}^{\perp}\right|_{s_{j}} ^{(k+1)}= & \left\{0.5\left|\mathcal{V}^{\perp}\right|\left(\frac{1}{|\widetilde{\psi}|} \frac{\partial|\widetilde{\psi}|}{\partial r}\right)\left(r_{j}-r_{i}\right)\right. \\
& \left.-0.5 \delta t \mathcal{V}^{\perp}\left(\mathcal{V} \cdot \frac{1}{|\widetilde{\psi}|} \nabla|\widetilde{\psi}|\right)-0.5 \delta t \mathcal{V}^{\perp}(\nabla \cdot \mathcal{V})\right\}\left.\right|_{s_{j}} ^{(k)},
\end{aligned}
$$

where $s_{j}$ denotes the edge centre and $r$ refers to the parametric description of the edge $r(\lambda)=r_{i}+\lambda\left(r_{j}-r_{i}\right) ; \quad \lambda \in[0,1]$.

\subsection{MPDATA based error indicators}

The above summarised evaluation of the pseudo-velocity (designated to compensate the leading truncation error of the first-order accurate upwind ad- 
vection scheme) naturally dictates error indicators for mesh refinement akin to the Richardson extrapolation. These indicators take advantage of the MPDATA property that more accurate solution is obtained from every subsequent upwind iteration. Because the first- and second-order solutions as well as the leading error are known explicitly regardless of the mesh adaptivity, such indicators impose minuscule computational complexity and overhead.

The concept of MPDATA based error indicators was proposed in [50] for hyperbolic problems in the context of compressible high-speed flows. The indicator relied on the magnitudes of the directional derivatives of the local solution error estimate, provided by the second term on the rhs of (12) for $k>1$. It was shown effective both for static and dynamic mesh adaptivity. Mathematically the PDEs (1) are close to PDEs governing incompressible viscous flows. For the latter practical indicators extensively used the energy norm, as it gives information on the accuracy achieved in each computational cell as well as globally [59]. Commonly, the energy norm is built on the element integrated product of the deviatoric stress and strain-rate errors. For the inviscid anelastic system (1) such a norm would vanish, as the deviatoric stress tensor is identically zero in (1). As there is no unique way to devise a practical error indicator [15], we take a freedom to delegate the role of the energy norm to the $L^{2}$ norm of the error fluxes in (12) taken over the local neighbourhood of each node. Arguably such a norm adopts the spirit of the energy norm, as it senses the momentum forcing via the auxiliary variable $\widetilde{\mathbf{u}}$, specified in (4), and effects akin to subgrid-scale stress through the ILES property of MPDATA.

Here, the implementation of the MPDATA based error indicator follows the standard strategy for static mesh refinement, whereupon the error indicator for the new mesh resolution is computed after the solution on the initial mesh is reached. In this implementation $\mathcal{K}=2$ and the second order solution for auxiliary variable $\widetilde{\psi}$ is taken from the MPDATA procedure (12). ${ }^{3}$ The corresponding truncation error fluxes are used to construct an error indicator as

$$
\epsilon_{i}=\|e\|_{i} / e_{m}
$$

where in line with (12) for $\mathcal{K}=2$

$$
\left(\|e\|_{i}\right)^{2}=\left(\frac{\delta t}{\vartheta_{i}}\right)^{2} \sum_{j=1}^{l(i)}\left[F_{j}^{\perp(2)}\left(\widetilde{\psi}_{i}^{(1)}, \widetilde{\psi}_{j}^{(1)}, \mathcal{V}_{j}^{\perp}(2)\right) S_{j}\right]^{2}
$$

To ensure that the error is equally distributed, the permissible value of the

$\overline{3}$ In all examples presented here we use the MPDATA infinite gauge option for which $\mathcal{K}=2$ by design; cf. section 5.1 in [42]. 
overall error for the computational domain is prescribed following [59], as

$$
e_{m}=\eta\left(\frac{1}{N} \sum_{i=1}^{N} \widetilde{\psi}^{(2)} \widetilde{\psi}^{(2)}\right)^{1 / 2}
$$

where $\eta$ is a desired specified percentage error (in this paper $\eta=0.05$ was used), and $N$ is the number of nodes in the primary mesh. Following the remarks in [60], we note that a loose interpretation of (18) is that it aims at achieving a specific percentage error in a global norm by approximately aiming at the RMS value of truncation error being some specified fraction of the RMS value of the exact solution approximated here by $\widetilde{\psi}^{(2)}$. A more intuitive use of the error density, in (20), has been studied in [30] and reported to be less effective.

The above procedure determines the magnitude of error indicator $\epsilon_{i}$ in every node of the initial mesh and allows to specify the desired size of new mesh resolution according to

$$
\delta x_{i}^{\text {new }}=\delta z_{i}^{\text {new }}=\delta x_{i}^{\text {old }} / \epsilon_{i}
$$

Additionally, we impose practical upper and lower bounds on $\delta x_{i}^{\text {new }}$ and $\delta z_{i}^{\text {new }}$. In our implementation, a new dual mesh is generated from a triangular primary mesh obtained by means of the advancing front technique with the mesh resolution controlled in the background mesh according to (21).

The adopted mesh refinement approach aims for an optimal mesh with the error similar in every cell; however, the construction of the error indicator is by no means unique. Various combinations of the auxiliary variables $\widetilde{\psi}$ in (1) and their corresponding truncation errors were explored in this study for their use in the error indicator. For the gravity wave problems addressed here the best candidate found is the vertical velocity component - unsurprisingly, as it is the dependent variable that directly reflects nonhydrostacy of stratified flows. We have also explored substituting (19) with the norm emphasising the solution error, rather than the error flux,

$$
\left(\|e\|_{i}\right)^{2}=\sum_{j=1}^{l(i)} 0.25\left(\delta \widetilde{\psi}_{i}^{(1)}+\delta \widetilde{\psi}_{j}^{(1)}\right)^{2},
$$

where $\delta \widetilde{\psi}$ is a shorthand for the second term on the rhs of (12). However, for the applications addressed, this indicator was causing minor local over-refinement that had to be moderated by adjusting the specified percentage error in (20). The impact of using (22) instead of (19) was consequently overshadowed by the related amendments to the specified percentage error, and in practice the 
error indicator (19) produced slightly better quality of meshes with smoother changes in spatial resolution.

\subsection{Static mesh refinement for a two-scale mountain wave}

The slab-symmetric benchmark problem of Schär et al [34] is used to demonstrate the implementation of MPDATA based error indicator and mesh adaptivity. The problem is designed to expose issues of horizontal resolution requirements for adequate geometrical representation of small scale topography. The selected problem parameters favour bifurcation into a qualitatively incorrect solution. The benchmark consists of an anelastic flow past a terrain profile under stably stratified atmospheric conditions, as described in [56] while following $[34,20]$. The terrain profile is Gaussian with superposed small-scale structure,

$$
z_{s}(x)=z_{s 0} \exp \left(\frac{-x^{2}}{a^{2}}\right) \cos ^{2}\left(\frac{\pi x}{\lambda}\right)
$$

where $z_{s 0}=0.25 \mathrm{~km}, a=5 \mathrm{~km}$ and $\lambda=4 \mathrm{~km}$. The buoyancy frequency $N=0.01 \mathrm{~s}^{-1}$; the ambient wind profile $u_{e}=U=10 \mathrm{~ms}^{-1}$ and $v_{e}=w_{e}=0$; the computational domain is $70 \times 21 \mathrm{~km}$ and the simulation time 5 hours. The upper $11 \mathrm{~km}$ of the model domain is designated as an absorbing layer, to simulate an infinite atmosphere and to suppress spurious wave reflection from the upper rigid-lid. Lateral absorbers extend for $10 \mathrm{~km}$ away from the boundaries.

The solution of the anelastic system (1) is obtained using the NFT model outlined in section 2. The initial flow is potential, determined by mass continuity and the boundary conditions. The lower boundary is impermeable, whereas at the top and the lateral boundaries $\left(\mathbf{u}-\mathbf{u}_{e}\right) \cdot \mathbf{n}=0$ is assumed; $\mathbf{n}$ is the outward unit normal to the model boundaries. The gravity-wave absorbers near the lateral and upper boundaries attenuate the solution towards environmental conditions with the inverse time scale $\alpha$ that increases linearly from $\alpha=0$ at the distance 10 and $11 \mathrm{~km}$ from the lateral and the upper boundary, respectively, to $\alpha=1200^{-1} \mathrm{~s}^{-1}$ at the boundaries. While in this work meshes conforming to the physical topography are investigated, in $[34,20,56]$ the terrain-following coordinate transformations were studied.

The procedure outlined in section 3.2 is used to design an adequately refined mesh. In order to show that the static adaptivity can combine information obtained from various types of meshes, we generate the initial solution on a purposely coarse $81 \times 46$ structured mesh shown in Fig. 3 . The resulting horizontal and vertical resolutions $\delta x=875 \mathrm{~m}$ and $\delta z \approx 467 \mathrm{~m}$ are, respectively, 

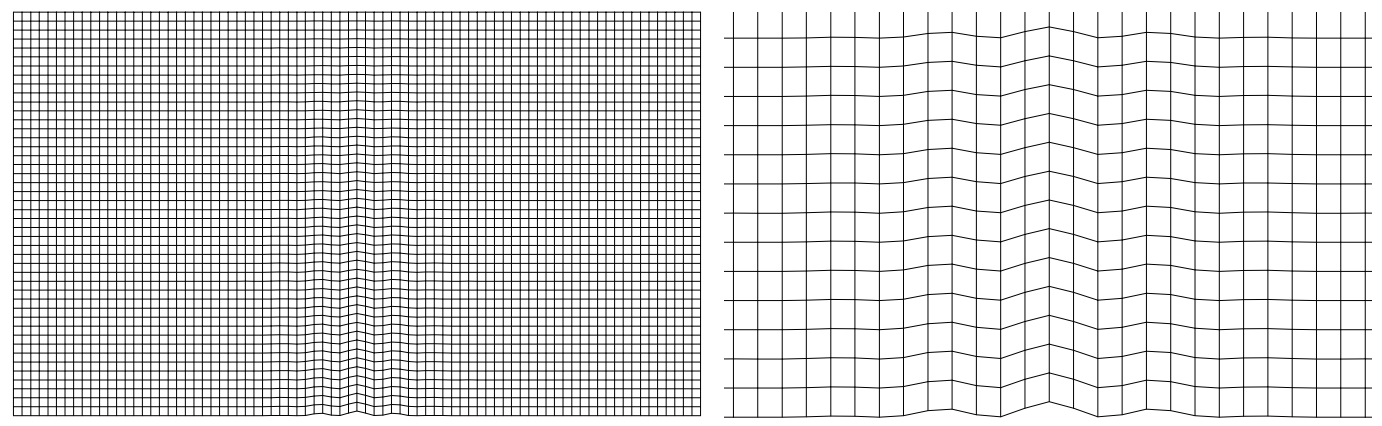

Fig. 3. Coarse structured mesh with underresolved topography (left panel), zoomed near the hill (right panel)

1.75 and 1.5 times larger than the increments used in the earlier works [34,56]. The mesh conforms to the terrain geometry and is constructed to mimic the standard Gal-Chen \& Somerville [13] terrain-following coordinates [44]. The resulting vertical velocity field, Fig. 4 left panel, evinces the characteristic spurious influence of the small-scale (nonhydrostatic) evanescent component of the solution on the large-scale hydrostatic-wave component characterised by deep propagation. However, these spurious effects are distinctively weaker than those shown in the corresponding Fig. 13a of [34], despite much coarser mesh used here. Interestingly, the result highlights different mechanisms in which too-coarse meshes can affect the solution. In this work the coarse mesh has too few points to adequately conform to the smooth geometry of the hill, as seen in the right panel of Fig. 3. The sharp changes in the lower boundary of the mesh give rise to the spurious flow features, but are relatively local. However, as shown in [34], in some methods relying on the coordinate transformation the spurious effects resulting from the inadequate representation of topography can propagate aloft. Nonetheless, the initial solution obtained even on such a coarse mesh carries sufficient information for the error indicator (18) to guide mesh refinement.
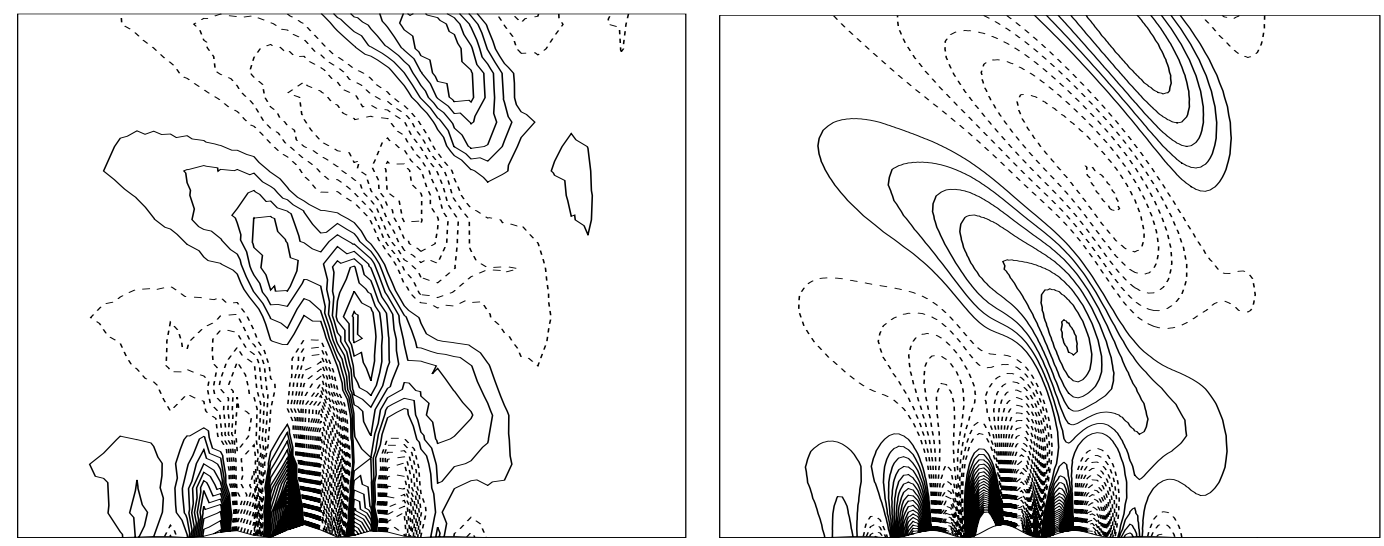

Fig. 4. Steady-state vertical velocity for the coarse structured mesh (left panel) and for the refined unstructured mesh; the contour interval is $0.05 \mathrm{~ms}^{-1}$ and zero contour lines are not shown. 


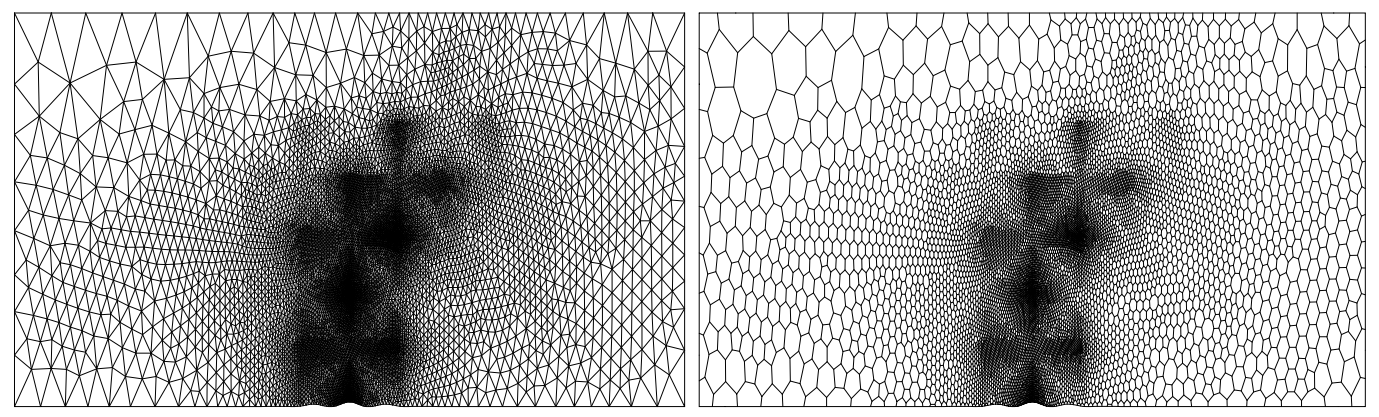

Fig. 5. Refined unstructured mesh; primary (left panel), dual (right panel).

In principle, the initial mesh could be used directly as a background mesh, however for a good quality of meshes it is usually beneficial when the background mesh is coarser than the initial mesh. Therefore, we generate a suitable alternative background mesh, consisting of larger triangular elements and interpolate linearly the desired new mesh resolution $(\delta x$ and $\delta z)$ to its nodes. Subsequently, the advancing front technique is employed for generating a new primary triangular mesh with the background spacing ranging from 170 to $3600 \mathrm{~m}$, shown in the left panel of Fig. 5, for which the corresponding dual mesh is shown in the right panel. As anticipated, the areas of finer mesh are present where the topography changes more rapidly and in the main wave train. In contrast, the mesh coarsens in the areas of smooth flow and absorbing boundaries. The right panel of Fig. 4 shows the corresponding vertical velocity field obtained with the adapted mesh. The new solution is obtained on a mesh containing 8830 nodes and is in agreement with the reference solution [34] and other published results [56] that used 10,011 grid points. Interestingly, the initial solution (not shown) obtained from a similarly coarse mesh but built on a base of a triangular primary mesh, results in similar adapted meshes and nearly identical final solution.

The solution obtained on the initial grid, containing 3726 nodes, used 1800 time steps with a constant interval $\delta t=10 \mathrm{~s}$, including computation of all geometrical metrics required by the dual mesh. The elliptic solver used on average six iterations (viz. evaluations of the generalised Poisson operator on the lhs of eq. 11) per time step, to reach $\varepsilon=\left\|\left(\delta t / \rho_{o}\right) \nabla \cdot\left(\mathbf{u} \rho_{o}\right)\right\|_{\infty}<10^{-5}$ convergence threshold, five orders of magnitude smaller than the maximal Courant number; see [38] for a discussion. ${ }^{4}$ The consumed CPU time was $68 \mathrm{~s}$. The corresponding solution on the adapted mesh containing 8830 nodes used 6000 time steps with $\delta t=3 \mathrm{~s}$ and four elliptic-solver iterations per time step, and took $464 \mathrm{~s}$ of CPU time.

\footnotetext{
$\overline{4}$ The same accuracy threshold was prescribed in all examples presented in this paper, and all computations were conducted in single precision on a single Intel(R)i7 975 processor.
} 


\section{Tetrahedral meshes; simulation of strongly stratified flow past a steep isolated hill}

In contrast to modelling engineering flows, where simulations on tetrahedral and related meshes have achieved a high degree of maturity, their applicability to atmospheric flows is still being explored. While prismatic meshes are ideally suited for global models - for which the (relatively) thin atmosphere imposes stringent constraints on the design of numerical models - the unstructured tetrahedral discretisation can benefit small- and mesoscale models. For example, applications involving terrain so complex as caves and canyons cannot be easily resolved with continuous mappings. Moreover, as the tetrahedral discretisation provides means for accommodating irregular interfaces, it provides new avenues for study of cloud physics. On the other hand, atmospheric flow simulations pose new challenges for tetrahedral discretisation, because the underlying hydrostatic balance and physics (e.g., rainfall and radiation) are predominantly ordered in the vertical direction. Here, we probe the accuracy of tetrahedral discretisation against prismatic and Cartesian meshes, in the context of strongly stratified flow past a steep 3D mountain. For this purpose, we adopt the canonical problem of a low-Froude number flow past an axially-symmetric hill $[18,11,37]$, simulated in [45] on dual meshes derived from structured and prismatic primary meshes.

The cosine hill defined as

$$
\begin{aligned}
& h(x, y)=h_{0} \cos ^{2}(\pi r / 2 L) \text { if } r=\left(\left(x-x_{0}\right)^{2}+\left(y-y_{0}\right)^{2}\right)^{1 / 2} \leq L \\
& h(x, y)=0 \text { otherwise }
\end{aligned}
$$

where the half-width $L=3000 \mathrm{~m}$ and height $h_{0}=1500 \mathrm{~m}$, is centred at the bottom of the computational domain. The domain size is $5 L \times 4 L \times 2 L$ in $x, y$ and $z$ directions, respectively. Two primary meshes consisting of tetrahedral elements were generated in the domain. The first one (not shown) using a uniform background point spacing of $\delta x=\delta y=\delta z=120 \mathrm{~m}$ giving 1945090 of the total number of points, and the second one with the varying point spacing. Figure 6 shows two cross-sections of the primary mesh that consists of 337510 points and uses varying resolution ranging from $450 \mathrm{~m}$ at the boundaries to about $100 \mathrm{~m}$ in the hill's vicinity. The number of points in the varying resolution tetrahedral mesh is not only substantially lower than in the constant resolution tetrahedral mesh but also lower than in the prismatic (refined in the horizontal only) and Cartesian meshes used for this problem in [45]. In order to produce solutions of matching quality, those meshes consisted of 692533 and 1121812 computational points respectively.

The governing equations (1) assume Boussinesq limit, with the constant potential temperature of the reference state $\Theta_{o}=300 \mathrm{~K}$ and the ambient state 



Fig. 6. The central, $y=0$, vertical cross-section (top) of the tetrahedral mesh; the nearby mesh points are projected on the cross-section for visualisation. The horizontal cross-section (bottom) of the mesh at the elevation $z=(1 / 3) h_{0}$.

characterised by constant buoyancy frequency $N=10^{-2} s^{-1}$ and uniform wind $U=5 \mathrm{~ms}^{-1}$. With the specified hill geometry, the ambient conditions result in a low Froude number, $F r=U / N h_{0}=1 / 3$, flow. Because $h_{0} / L \sim \mathcal{O}(1)$, the problem is essentially nonhydrostatic and can be compared to experimental results given in [18]. All results are shown after two advective time scales $T=L / U(t=1200 s)$ when the main features of the solution are already established. The initial condition is provided by the solution of the potential flow problem, with a gradient of the potential perturbation imposed on the ambient wind. While the boundary conditions are rigid in $x, y$ and $z$, the gravity-wave absorbers near the upper and lateral streamwise boundaries attenuate the solution toward ambient profiles with absorbing coefficient increasing linearly from zero at the distance $L / 2$ from the boundary to $150^{-1} \mathrm{~s}^{-1}$ at the boundary.

The flow patterns displayed in figures 7 and 8 are computed for the varying 

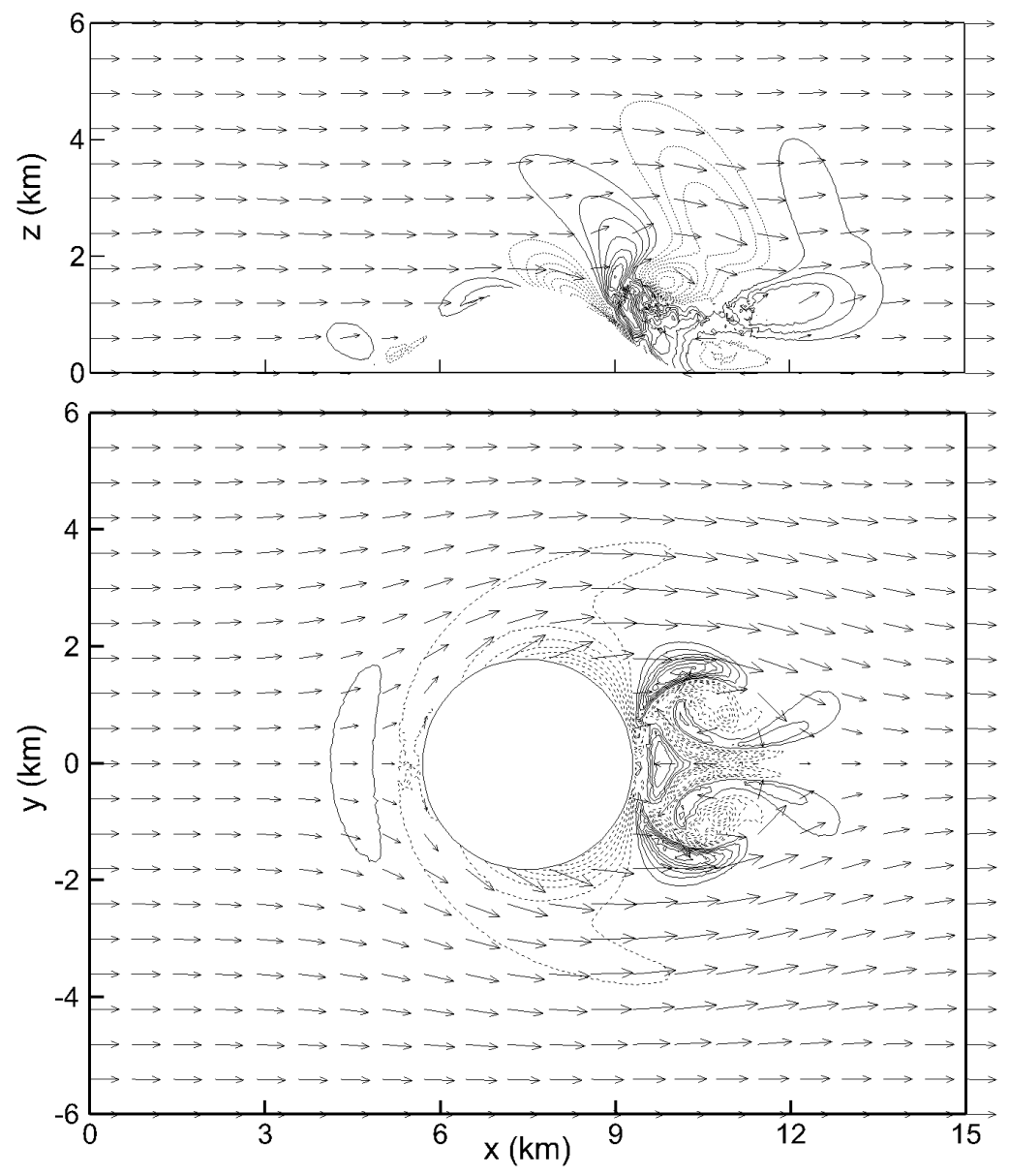

Fig. 7. $F r=1 / 3$ flow solution after two advective time scales $T=L / U$. Contours of vertical velocity in central $x z$ cross section $y=0$ (top) and in the $x y$ cross-section at $z=h_{0} / 3$ (bottom). The contour interval is $0.5 \mathrm{~ms}^{-1}$, and positive/negative contours are presented with solid/dashed lines; the zero contours are not displayed.

resolution mesh, ${ }^{5}$ and show key features of a low Froude number flow, including the characteristic separation and reversal of the lower upwind stream, and the formation of intense vertically-oriented vortices on the lee side of the hill $[18,37,11]$, with the flow aloft transitioning to the linear gravity wave response [35]. In Figure 7 , in the central $x z$ cross-section at $y=0$, a turbulent wake is formed in the lee-side of the hill and characteristic gravity waves response is visible above the wake. This result matches closely the reference solution obtained on structured and prismatic meshes (upper panels in Figs. 6 and 8 in [45]). Figure 7 , in $x y$ cross-section at $z=(1 / 3) h_{0}=500 \mathrm{~m}$, presents a pair of eddies behind the hill, showing the intrinsic three-dimensionality of the leeside flow. In brief, the reason of this flow structure is that the incoming flow up to $z_{c} \approx(1-F r) h_{0}$ — the so called dividing streamline [18] — is forced

$\overline{5}$ For plotting purposes in all 3D experiments, scalar fields are interpolated linearly to $2 \mathrm{D}$ Cartesian grids placed on the cross sectional planes cutting through the $3 \mathrm{D}$ fields. 


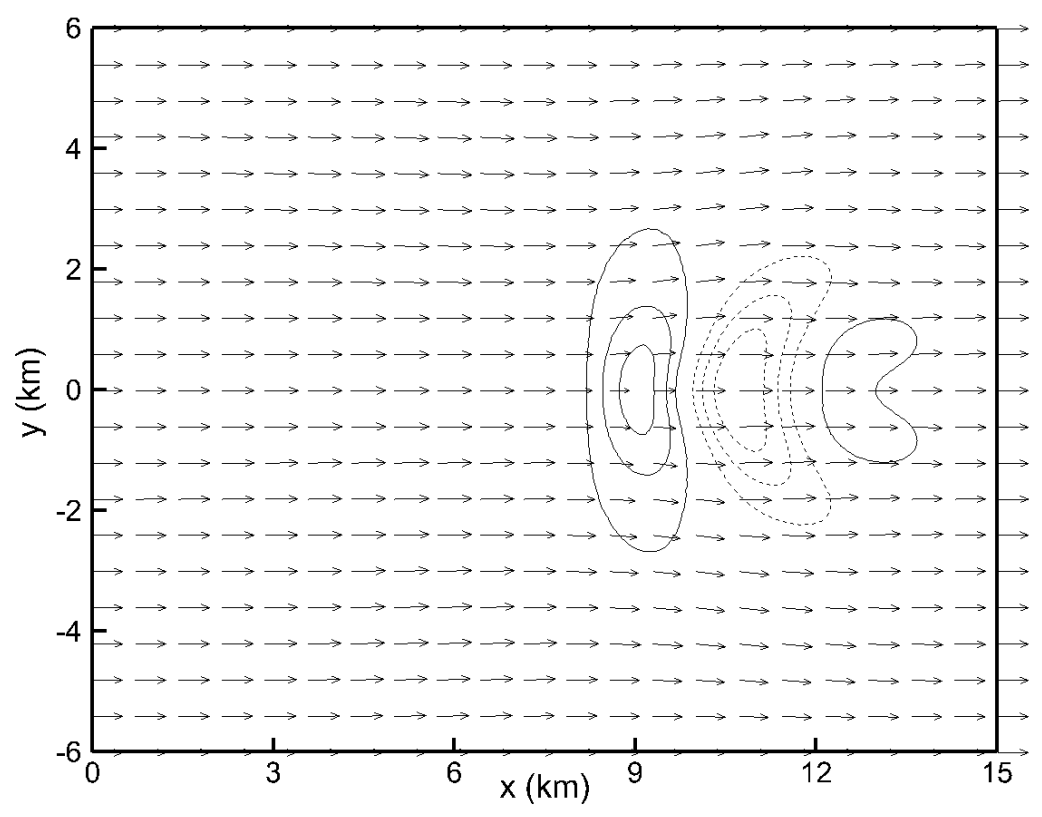

Fig. 8. As in the bottom panel of Fig. 7 but at $z=(5 / 3) h_{0}$ horizontal cross-section.

to deflect and split around to the hill as it lacks sufficient kinetic energy to go over the hill. Above the dividing streamline the hill is sufficiently low for the flow to go over, thus resulting the characteristic gravity response aloft. Figure 8 shows the horizontal structure of the gravity wave for the $x y$ crosssection at $z=(5 / 3) h_{0}=2500 \mathrm{~m}$. The corresponding solutions for the regular tetrahedral mesh are not shown, because their departures from the results in Figs. 7 and 8 are insignificant. The two horizontal flow patterns also compare well with the reference results in [45].

The statistics of the results are presented in Tables 1 and 2 for the tetrahedral meshes with constant and varying resolution, respectively. In the tables, the units of the three velocity components are in $\mathrm{ms}^{-1}$ and the potential temperature in K. The statistics of $\Theta^{\prime}$ are converted to the vertical displacements of isentropes using a crude approximation

$$
\eta_{z} \approx-\Theta^{\prime} \frac{g}{N^{2} \Theta_{o}} \approx-333 \Theta^{\prime} \mathrm{m}
$$

The statistics of dependent-variable fluctuations about the ambient state are evaluated over the entire computational domain. The irregularity of the mesh is taken into account when calculating the averages and standard deviations of the fluctuations. With $\vartheta_{i}$ denoting the cell volume surrounding mesh point 
$i$, the average values $\overline{\psi^{\prime}}$ are calculated according to

$$
\overline{\psi^{\prime}}=\frac{\sum_{i=1}^{n}\left(\psi_{i}^{\prime} \vartheta_{i}\right)}{\sum_{i=1}^{n} \vartheta_{i}},
$$

and the corresponding standard deviations $\sigma_{\psi^{\prime}}$ are calculated as

$$
\sigma_{\psi^{\prime}}=\frac{\sum_{i=1}^{n}\left(\left(\psi_{i}^{\prime}-\overline{\psi^{\prime}}\right)^{2} \vartheta_{i}\right)}{\sum_{i=1}^{n} \vartheta_{i}}
$$

where $n$ denotes the total number of nodes in the domain. The statistics for the two tetrahedral meshes as well as those provided for the refined prismatic mesh (Table 2 in [45]) are in close agreement. ${ }^{6}$

\begin{tabular}{|l|l|l|l|l|}
\hline Variable & Maximum & Minimum & Average & Standard Deviation \\
\hline$u^{\prime}$ & 3.51 & -11.39 & 0.06 & 0.76 \\
\hline$v^{\prime}$ & 8.04 & -8.48 & $4.7 \times 10^{-6}$ & 0.47 \\
\hline$w^{\prime}$ & 5.35 & -4.80 & $-1.02 \times 10^{-4}$ & 0.36 \\
\hline$\Theta^{\prime}$ & 2.85 & -1.48 & $-4.7 \times 10^{-3}$ & 0.20 \\
\hline$\eta^{\prime}$ & -949 & 493 & 1.57 & 66.6 \\
\hline
\end{tabular}

Table 1

Fluctuations' statistics on a tetrahedral mesh with constant resolution

\begin{tabular}{|l|l|l|l|l|}
\hline Variable & Maximum & Minimum & Average & Standard Deviation \\
\hline$u^{\prime}$ & 3.42 & -11.33 & 0.06 & 0.78 \\
\hline$v^{\prime}$ & 8.42 & -8.26 & $1.93 \times 10^{-6}$ & 0.48 \\
\hline$w^{\prime}$ & 5.56 & -4.76 & $-6.40 \times 10^{-5}$ & 0.36 \\
\hline$\Theta^{\prime}$ & 2.93 & -1.52 & $-6.99 \times 10^{-3}$ & 0.20 \\
\hline$\eta^{\prime}$ & -976 & 506 & 2.33 & 66.6 \\
\hline
\end{tabular}

Table 2

Fluctuation's statistics on a tetrahedral mesh with varying resolution

The solution obtained on the mesh with varying resolution, containing 337510 nodes, used 960 time steps with a constant $\delta t=1.25 \mathrm{~s}$ and required 14 iterations of the elliptic solver per time step. The resulting CPU time of the

$\overline{6}$ The $u_{e}=5 \mathrm{~ms}^{-1}$ was not subtracted from the values of $u$ given in [45]. 
Intel processor (recall footnote 4) was $15204 \mathrm{~s}$. The corresponding solution on the regular tetrahedral mesh, containing 1945090 nodes, used the same time interval and the same number of the elliptic solver iterations, but it required 87713 s of CPU time. Computations using the same model and time interval were repeated with identical Cartesian and prismatic meshes to those used in [45] and showed that CPU time changes almost proportionally to the number of nodes in a mesh, for all types of investigated meshes. This was expected since all these meshes have a similar average number of edges per node. A further assessment of the overall economy of models using $i, j, k$ and edge-based data structures, for this particular benchmark, is included in [45].

\section{Meshes with highly variable resolution; simulation of critical- level orographic flows}

Sections 3 and 4 examined the efficacy of variable-resolution flexible meshes for simulating orographically forced gravity-wave response in canonical media with uniform wind and constant stratification. However, in the atmosphere, wind and stratification generally vary with height, and this can profoundly affect the response. This is particularly evident for ambient flows with critical levels, identified by the horizontal wave phase speed equal to the ambient velocity component in the direction of the wave propagation. In steady mountain waves like those discussed in sections 3 and 4 the phase speed is zero, so the critical level is where the ambient wind vanishes. The influence of the critical levels on the gravity waves propagation is widely discussed in the literature; see $[2,9,28,16,8,57]$ and references therein. Theoretically, as the wave packet approaches a critical level the group velocity and the vertical wavelength tend to zero, while the horizontal velocity grows unboundedly, and the effective wave absorption occurs below the critical level (i.e., within the critical layer). This singularity of the linear predictions indicates that processes acting on small scales become important in critical layers; in particular, the nonlinear steepening and overturning of the waves. In $3 \mathrm{D}$, this is a challenge for structured grids, which require uniformly high resolution to gain insights into the morphology of critical layers.

Here, the anelastic equations (1) are integrated on highly anisotropic and inhomogeneous mesh with high resolution concentrated in a small portion of the computational domain. The geometry of the problem and flow conditions are selected to reproduce the numerical experiment defined in [16]. The domain is $100 \times 100 \times 3 \mathrm{~km}^{3}$, with an axially symmetric mountain

$$
h(r)=h_{0}\left(1+\frac{r^{2}}{a^{2}}\right)^{-3 / 2}, r \equiv \sqrt{x^{2}+y^{2}}
$$


centred at the bottom of the domain. The ambient velocity profile with a reverse linear shear is defined as

$$
U(z)=U_{0}\left(1-\frac{z}{z_{c}}\right)
$$

where $z_{c}$ denotes the altitude of the critical level. Free-slip impermeable boundary conditions are applied at the vertical and spanwise lateral boundaries. At the streamwise lateral boundaries the ambient profile is assumed. The absorbing layers are employed in the vicinity of the upper and streamwise lateral boundaries with respective thickness of 1 and $10 \mathrm{~km}$. The inverse timescale of the absorbers is $150^{-1} \mathrm{~s}^{-1}$ at the boundaries.

For this problem we employ a primary unstructured mesh built from distorted prisms with triangular bases. This is because the computational domain is relatively thin in the vertical, and the use of prismatic mesh allows a considerably finer resolution in the vertical direction. To construct such a mesh, firstly, a planar triangular mesh is generated that defines the $x$ and $y$ coordinates for all nodes. Secondly, the layers of prisms are stack in the vertical, such that the vertical position of the node is specified as

$$
\tilde{z}_{i, k}=\tilde{z}_{i, k-1}+\delta \tilde{z}_{k}
$$

Here, $i=1,15059$ numbers the nodes in each prismatic layer, $k=1,70$ numbers the layers, $\tilde{z}_{i, k}$ is the height of the layer with respect to the flat bottom, $\delta \tilde{z}_{k}$ is the increment of the $k$ th layer, with $\tilde{z}_{i, 0}=0$ and $\delta \tilde{z}_{1}=0$. Finally, the layers are elevated according to the shape of the mountain by displacing $z$ coordinate of the mesh to mimic the terrain-following coordinate transformation $[13,56]$

$$
z_{i, k}=\tilde{z}_{i, k}\left(1-\frac{h_{i}}{H}\right)+h_{i}
$$

where $h_{i}$ is the height of the mountain at the $i$ th node in each layer and $H=3 \mathrm{~km}$ is the top of the model domain. The horizontal grid size is approximately $150 \mathrm{~m}$ in the vicinity of the hill and gradually grows larger to be around $3.5 \mathrm{~km}$ near the boundary of the domain. To ensure adequate geometry representation, a finer point resolution is applied where the mountain slope is higher. The vertical increment $\left(\delta \tilde{z}_{k}\right)$ grows gradually from $30 \mathrm{~m}$ close to or below the critical level to $100 \mathrm{~m}$ near the top of the domain. Figures 9 and 10 show, respectively, a representative primary surface mesh for the lateral and top surfaces of the model the domain, and a fragment of the bottom-surface mesh conforming to the hill. 


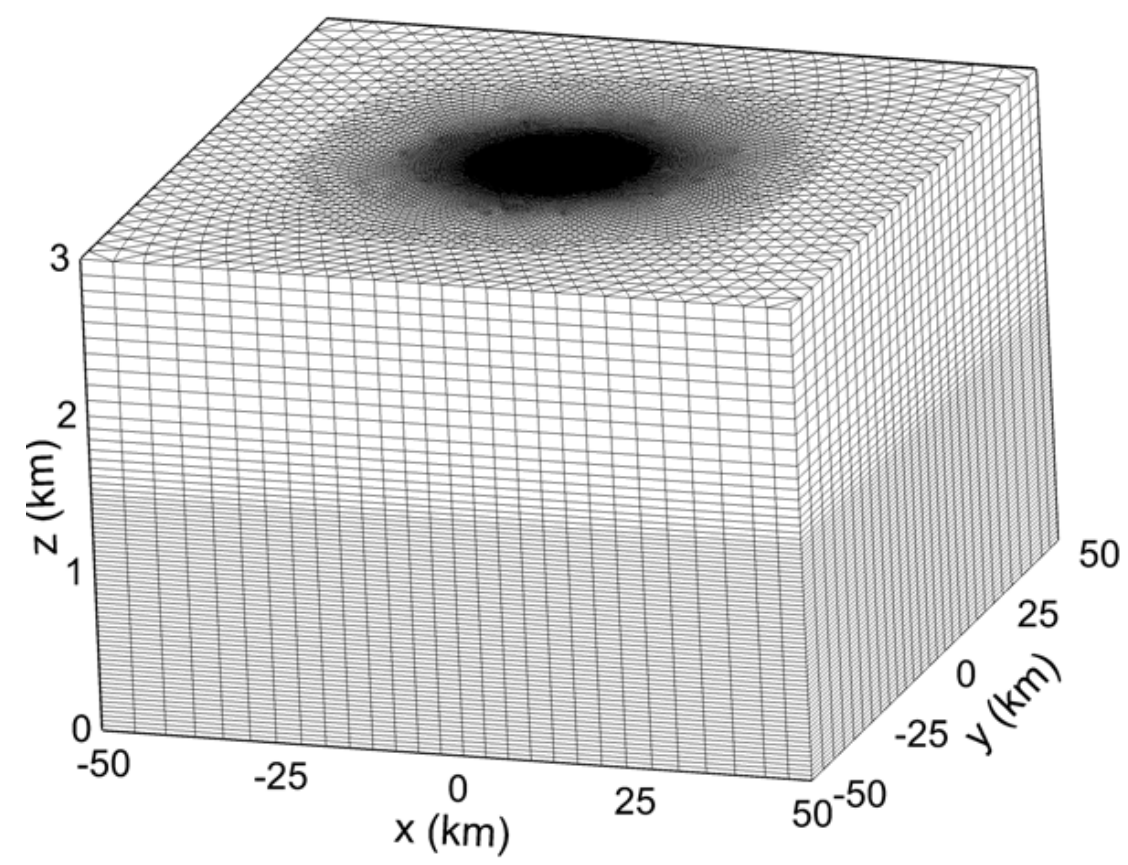

Fig. 9. The surface mesh of the prismatic volumetric mesh.

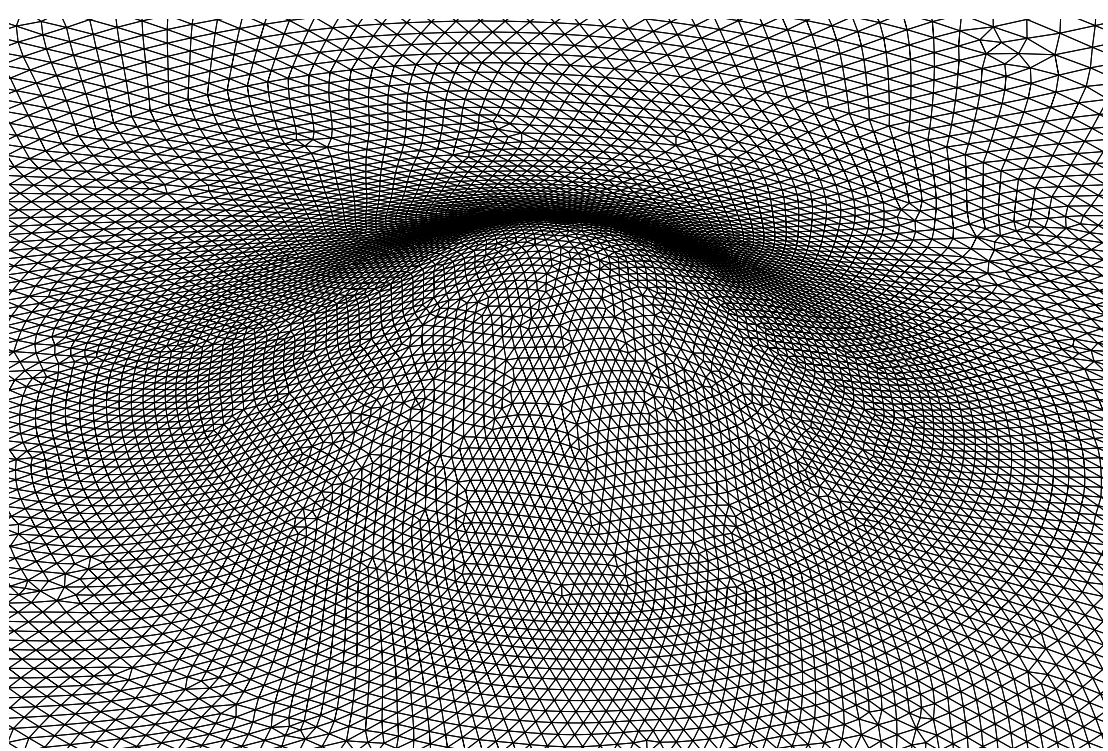

Fig. 10. A zoomed fragment of the horizontal triangular surface mesh at the bottom of the domain.

Following [16], the ground-level wind speed $U_{0}=10 \mathrm{~ms}^{-1}$, the buoyancy frequency $N=0.01 \mathrm{~s}^{-1}$, the half-width of the hill $a=5000 \mathrm{~m}$, and the position of the critical level $z_{c}=1000 \mathrm{~m}$. Consequently, the ratio of the horizontal to the vertical wave number of the hydrostatic mountain wave forced by the hill is $U_{0} / N a=0.2$ and the Richardson number $R i=\left(N z_{c} / U_{0}\right)^{2}=1$. The ambient shear flow is thus hydrostatic and stable. Four key benchmarks for different mountain heights have been selected from those studied in [16]: their LS2 for a linear case; and LS3, LS4 and LS5 for nonlinear cases. The term "linear" or 


\begin{tabular}{|l|l|l|l|l|l|}
\hline Experiment & $\mathrm{Ri}$ & $\hat{h}$ & $D / D_{0}(6)$ & $D / D_{0}(10)$ & $D / D_{0}(18)$ \\
\hline LS2 (linear) & 1 & 0.05 & 0.923 & 0.954 & 0.997 \\
\hline LS3 (nonlinear) & 1 & 0.1 & 0.983 & 1.02 & 1.160 \\
\hline LS4 (nonlinear) & 1 & 0.2 & 1.04 & 1.15 & 1.26 \\
\hline LS5 (nonlinear) & 1 & 0.3 & 1.09 & 1.22 & 1.23 \\
\hline
\end{tabular}

Table 3

Parameters of the selected numerical experiments, LS2-LS5 after [16].

"nonlinear" is read from Fig. 8 in [16]. The magnitudes of a nondimensional mountain height $\hat{h}=h_{0} N / U_{0}$ (viz. the inverse Froude number) are specified by varying $h_{0}$. All four experiments summarised in Table 3 were run until the dimensionless time $T=t U_{0} / a=18$. The mountain wave drag $D(T)$ is calculated numerically as $D=-\int_{\Gamma} p^{\prime} n_{x} d S$, where $\Gamma$ denotes the mountain surface, and $n_{x}$ is the $x$ component of the unit vector normal to $\Gamma$. The numerical drag $D$, listed in the table at $T=6,10$ and 18 , is normalised by the analytic hydrostatic drag for uniform ambient flow, $D_{0}=\frac{\pi}{4} \rho_{0} N U_{0} a h_{0}^{2}$ [35].

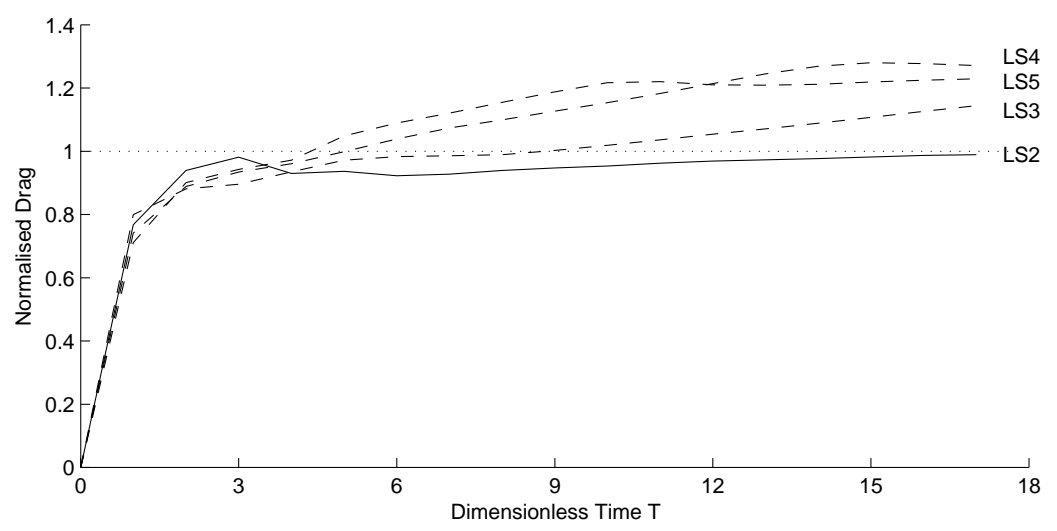

Fig. 11. Normalised drag history. The linear case is shown in solid line, while nonlinear cases are in dashed lines.

Figure 11 shows the histories of normalised drag, corresponding to those in Fig. 9 of [16] for the LS2-LS5 experiments. It should be noted that in [16] all experiments were initialised by growing the mountain gradually over the first $T=8$ time units; whereas in the present study, the initial conditions assume ambient wind with a potential perturbation imposed to satisfy the mass continuity equation in (1) together with the boundary conditions on the fixed full-size mountain. In consequence, our results are substantially advanced in the dimensionless time compared to [16], and the details of this advancement depend on the hill size. These are of minor importance for the linear case that approaches a steady state, but are more consequential for the nonlinear cases 
that depart steadily from the linear results. Overall, the drag histories in Fig. 11 reproduce the dependence of the drag on the amplitude of the lowerboundary forcing reported in [16].
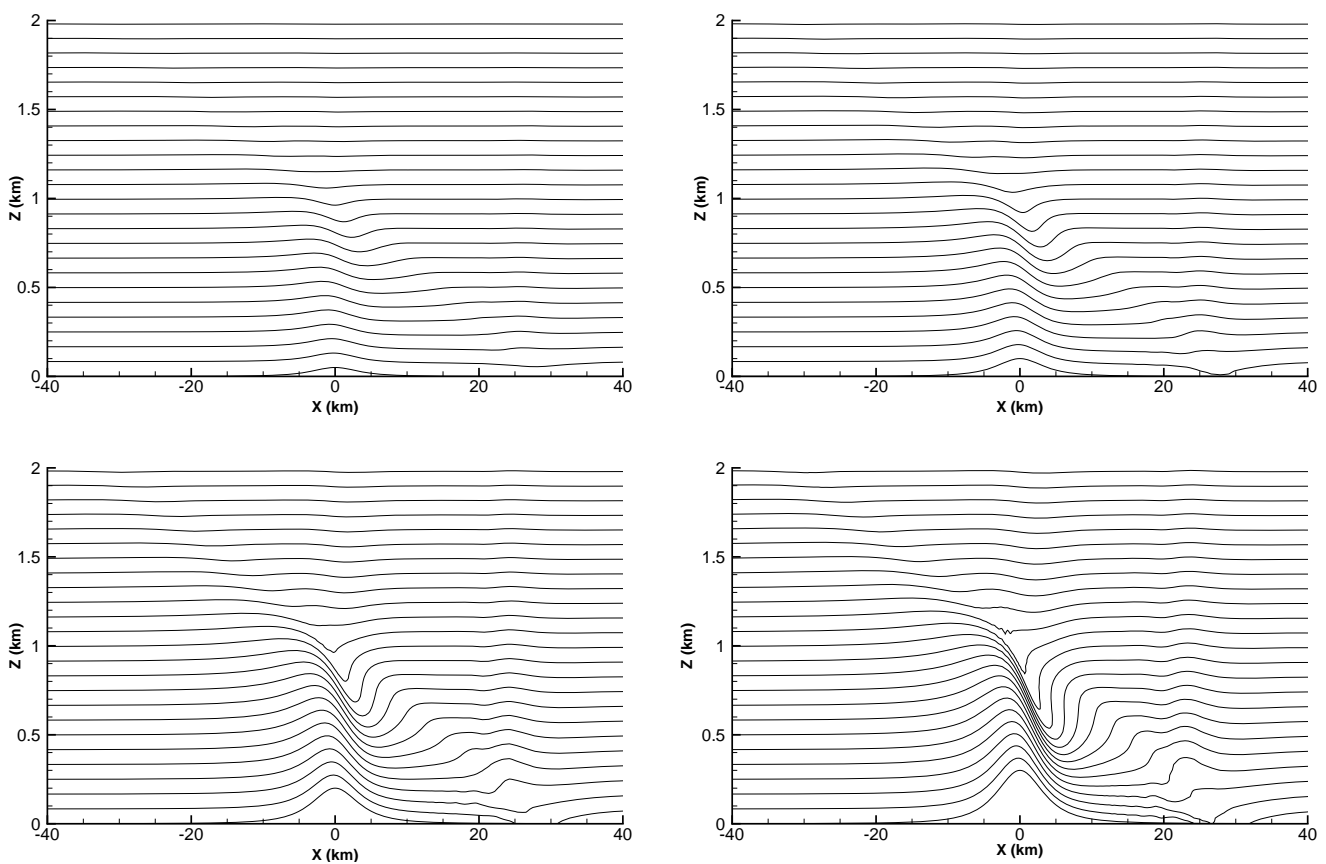

Fig. 12. Isentropes at $\mathrm{T}=6$ in $y=0$ vertical plane for experiments LS2 (left, top), LS3 (right, top), LS4 (left, bottom) and LS5 (right, bottom).

Figure 12 shows the isentropes in the $y=0$ vertical plane at $T=6$ for LS2-LS5 experiments. These solutions compare well with Fig. 13(a) and Figs. 16(a), (c), (d) at $T=18$ in [16]. As anticipated, they show that there is no significant propagation of the wave energy throughout the critical level. Furthermore, for sufficiently large amplitude of incoming waves nonlinearity dominates viscous (ILES) processes in the layer. This results in the wave steepening and overturning beneath the critical layer. However, the flow below the critical layer remains essentially decoupled from the flow aloft. Figure 13 highlights the dependence of the solution on the magnitude of the lower boundary forcing and time; it corresponds to Fig. 12 with the results displayed at the later time $T=18$. Comparing the corresponding panels in Figs. 13 and 12 shows that the flow stability diminishes with increasing amplitude of the mountain wave. Furthermore, it demonstrates that given a sufficiently long time and low viscosity even the "linear" wave overturns right beneath the critical layer. The top two panels in Fig. 13 show solutions qualitatively similar to their earlier forms, with the one on the right transitioning to turbulence but still decoupled from the flow aloft. This is corroborated in Fig. 14 that complements these two panels with the display of streamlines that capture the characteristic Kelvin's "cat's eye" circulation [28] in LS2 run but becoming irregular in LS3 experiment. In contrast, the two bottom panels of Fig. 13 already evince massive wave breaking with turbulent flow in the lee and evanescent perturbations, 
excited by convective eddies, penetrating through the critical layer. Notably, these results may be viewed as a 3D paraphrases of the 2D flow discussed in section 5 of [43].
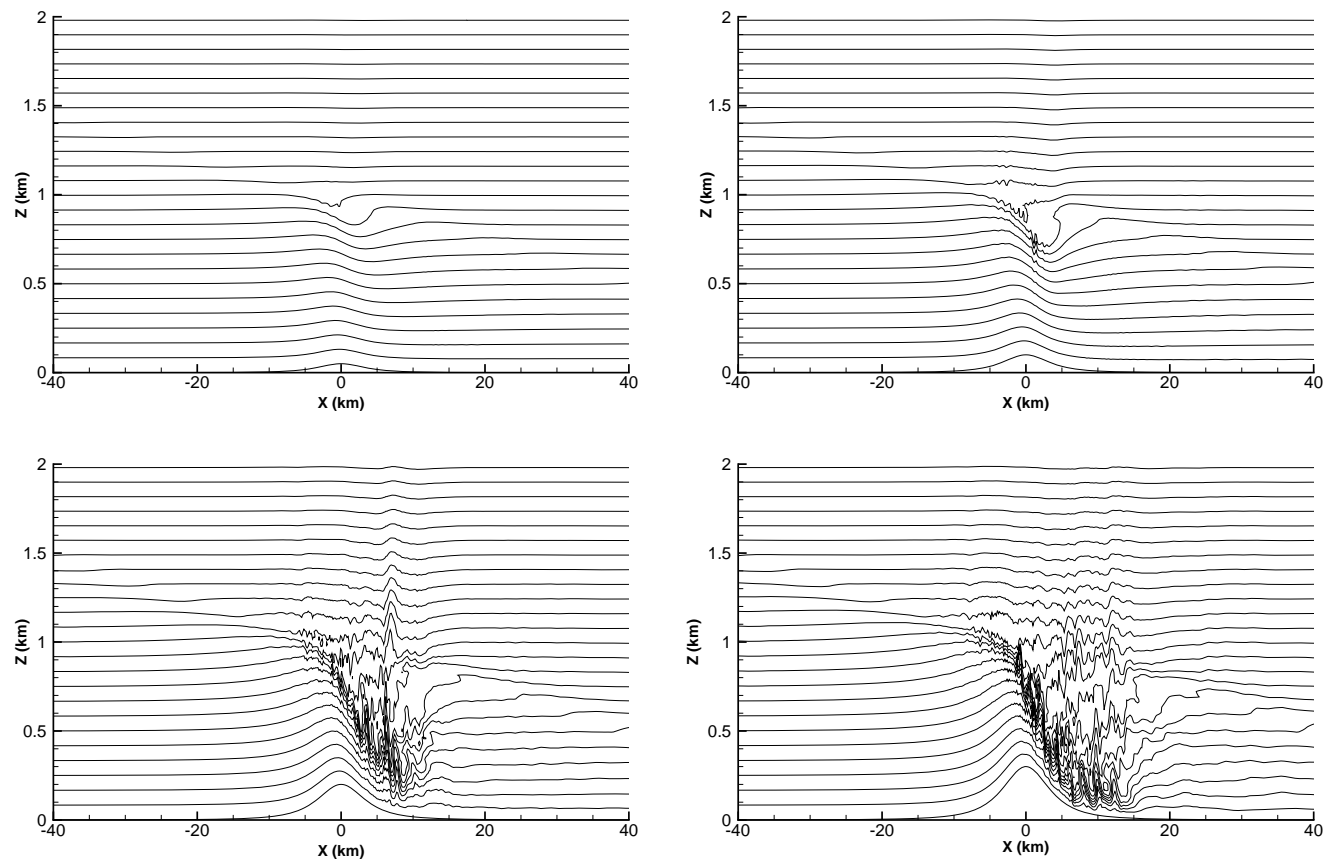

Fig. 13. As in Fig. 12 but at $\mathrm{T}=18$.
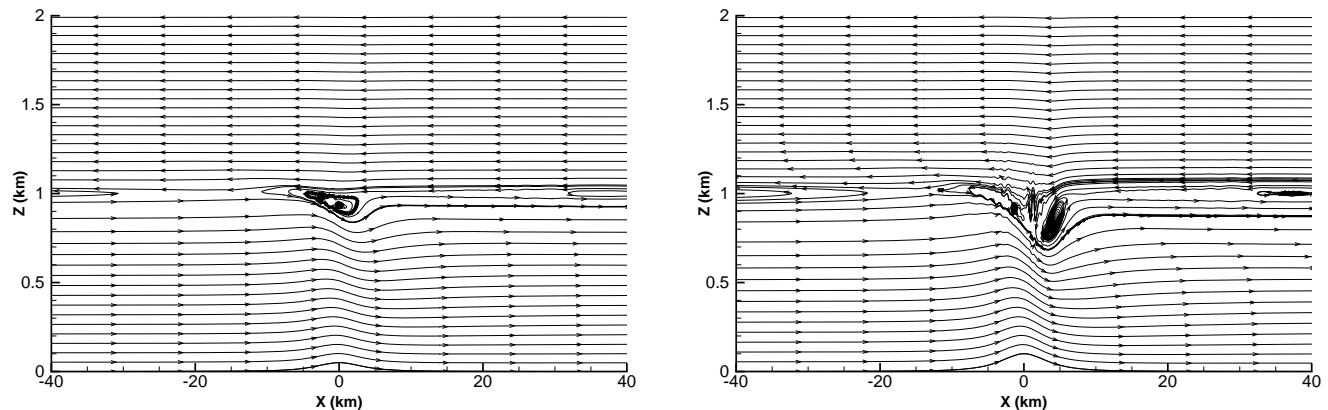

Fig. 14. Streamline corresponding to the isentrope display in the upper two panels of Fig. 13.

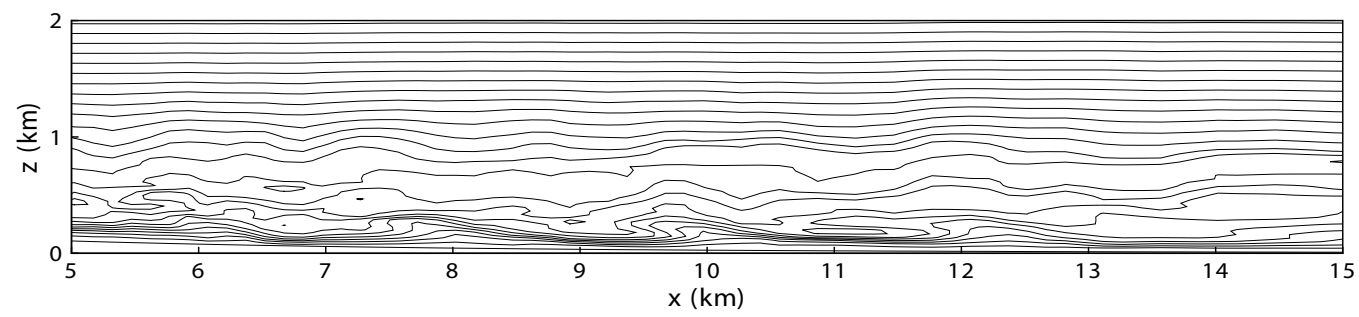

Fig. 15. A zoomed fragment of the isentrope display in the lower left panel of Fig. 13. 

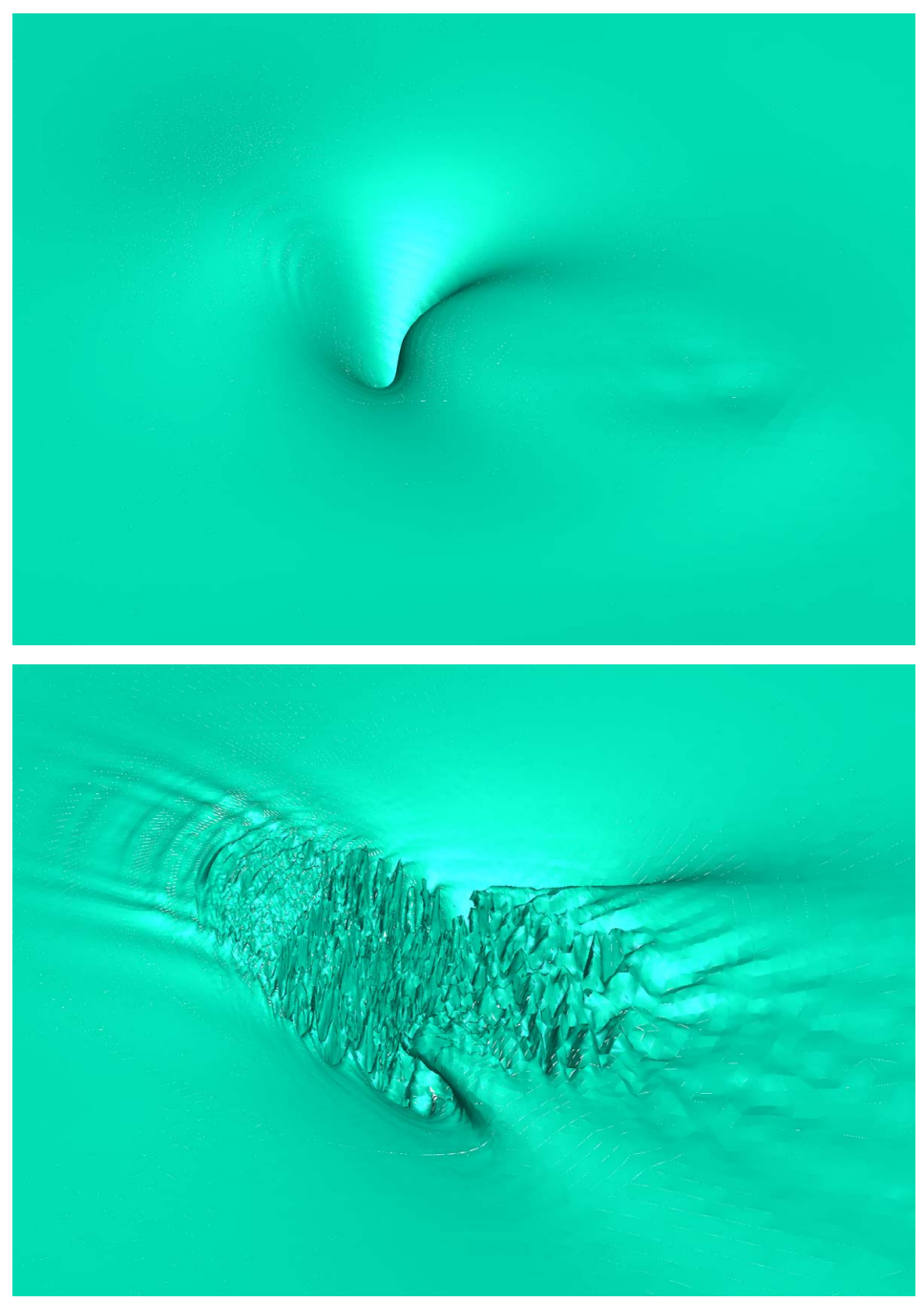

Fig. 16. A fragment of the isentrope with undisturbed height $z=0.94 z_{c}$ at $T=6$ (top) and $T=18$ (bottom) for the run LS5.

At the first glance, the physical content of the two bottom panels of Fig. 13 may seem dubious, because of the noisy appearance of the isentropes. However, this due to the heavily distorted display of the of the $80 \times 2 \mathrm{~km}^{2}$ vertical plane, in which these fine-scale features are resolved with 33 cells per $5 \mathrm{~km}$ of the horizontal distance. For substantiation, Fig. 15 shows a small, properly scaled fragment of the the right bottom panel of Fig. 13. Figure 16 shows a fragment of the isentropic surface, ${ }^{7}$ defined by its undisturbed height $z=0.94 z_{c}$, at the dimensionless times $T=6$ and 18 for the LS5 experiment. At $T=6$, the top panel of Fig. 16 roughly corresponds to the first isentrope beneath $z=1 \mathrm{~km}$ in the bottom-right panel of Fig. 12. It supplements the latter with a highlight of the 3D structure of the overturning mountain wave. At $T=18$, the lower panel of Fig. 16 evinces an abundance of fine scale features reflecting

7 The image is distorted, with displayed length across the diagonal and the vertical depth of about $40 \mathrm{~km}$ and $0.25 \mathrm{~km}$, respectively. 
convective eddies in the turbulent wake forming in the lee; cf. Fig. 15 for an undistorted display. This nature of the solution substantiates two important aspects of this work. First, it attests to the utility of the flexible meshing admitting highly anisotropic and inhomogeneous varying resolution in regions of interest. Second, it illustrates the utility of ILES approach assuring nonlinearly stable solutions on variable meshes without the need for explicit subgrid-scale (SGS) models. Noteworthy, high resolution is required to capture intermittent turbulence in the lee of the mountain, but variable resolution complicates explicit SGS modelling already at the theoretical level [27]. In contrast, ILES naturally selects the variable filter size and imposes no dissipative stability constraints controlled by the square of the local resolution.

The computations presented in this section typically required 10 iterations of the elliptic solver time step and $20924 \mathrm{~s}$ of the Intel processor CPU time per 1000 time steps.

\section{Conclusions}

A methodical study has been summarised that explores the flexibility and practicality of the finite volume discretisation operating on fully unstructured and adaptive meshes with the efficient edge-based data structure. The performance and potential of such meshes for modelling stably-stratified nonhydrostatic atmospheric flows past complex terrain were illustrated and quantified with challenging numerical simulations. In particular, 3D simulations of intricate nonlinear flows with a range of emerging scales were selected to probe the accuracy of finite volume discretisations on highly anisotropic and inhomogeneous meshes.

The limited-area nonhydrostatic nonoscillatory forward-in-time (NFT) model [45] has been extended with specialised static-mesh-adaptivity approach compatible with the model numerics. Namely, the error indicators for mesh refinement exploit inherent properties of the iterative MPDATA methods that underlie the model NFT integrators and as such impose minor computational complexity and overhead. Illustrative simulations using the static adaptivity for a challenging benchmark of a 2D stratified flow past a two-scale mountain [34] proved the approach sound and exemplified the potential for an automated generation of meshes with nodes distribution optimised to reflect flow features and the geometry of topography. The issue of small-scale topography and associated grid transformations is gaining importance due to the continued increase in horizontal resolution of operational weather prediction and climate models. This makes the options for complementary solutions using flexible meshing and alternative geometry representations attractive. 
The successful validation of the NFT MPDATA model has been further extended to meshes constructed from the irregular tetrahedral primary grids. The presented results for intricate strongly stratified flow past a steep isolated hill [18] confirm that the approach performs well for arbitrary shaped meshes. Furthermore, the obtained results match the accuracy attainable on Cartesian grids and prismatic unstructured (in the horizontal) meshes with a similar spatial resolution. Last but not least, special attention has been given to simulations of complex anisotropic and inhomogeneous flows with a range of scales emerging intermittently in the course of the simulations. Numerical study of such flows can clearly benefit from highly anisotropic and inhomogeneous meshes conforming to the nature of the flow. Our simulations of sheared stratified 3D orographic flows with critical-level [16] document good accuracy offered by, while illustrating cognitive benefits of, prismatic unstructured-mesh discretisation with high degree of the mesh variability.

Similarly like in engineering, a mesh type preference for atmospheric flows depends on the specific problem and the modeller's focus of interest. A fully unstructured mesh generation is more involved than a straightforward specification of a regular Cartesian grid. However, an automatic procedure for an unstructured mesh generation can be established and made user friendly for a given class of problems and a subsequent routine usage. The required skill and effort would naturally increase for unstructured mesh generation in solutions of new problems and research studies that involve extensive experimentation with optimal mesh resolution. Interestingly, because finite-volume models capable of producing quality solutions on unstructured meshes can also be run on regular grids $[52,44]$, variable mesh resolution only adds value to computational meteorology at a moderate price. Following experience from other fields, new capabilities offered by unstructured-mesh methods are likely to complement rather than replace established atmospheric models operating on regular grids.

\section{Acknowledgements:}

Comments from Christian Kühnlein and two anonymous referees helped to improve the paper. The authors acknowledge support by the NERC/G004358 award and funding received from the European Research Council under the European Union's Seventh Framework Programme (FP7/2012/ERC Grant agreement no. 320375). 


\section{References}

[1] D.P. Bacon, N.N. Ahmad, Z. Boybeyi, T.J. Dunn, M.S. Hall, P.C.S. Lee, R.A. Sarma, M.D. Turner, K.T. Waight, S.H. Young, J.W. Zack, A Dynamically Adapting Weather and Dispersion model: The Operational Multiscale Environment Model with Grid Adaptivity (OMEGA), Month. Weather Rev. 128 (2000) 2044-2067

[2] J.R. Booker, F.P. Bretherton, The critical layer for internal gravity waves in a shear flow. J. Fluid Mech. 27 (1967) 513-539.

[3] C.J. Budd, M.J. Cullen, E.J. Walsh, Monge-Ampere based moving mesh methods for numerical weather prediction, with applications to the Eady problem, J. Comput. Phys. 236 (2013) 247-270.

[4] C.J. Budd, R.D. Russell, E. Walsh, The geometry of r-adaptive meshes generated using optimal transport methods, J. Comput. Phys. 282 (2015) 113137.

[5] Bunge H-P, M.A. Richards, and J.R. Baumgardner, A sensitivity study of threedimensional spherical mantle convection at $10^{8}$ Rayleigh number: Effects of depth-dependent viscosity, heating mode, and an endothermic phase change, J. Geophys. Res. 102 (1997) 11,991-12,007

[6] T.L. Clark, R.D. Farley, Severe downslope windstorm calculations in 2 and 3 spatial dimensions using anelastic interactive grid nesting - a possible mechanism for gustiness, J. Atmos. Sci. 41 (1984), 329-350.

[7] J.A. Domaradzki, Z. Xiao, P.K. Smolarkiewicz, Effective eddy viscosities in implicit large eddy simulations of turbulent flows, Phys. Fluids 15 (2003) 38903893.

[8] A. Dörnbrack, J.D. Doyle, T.P. Lane, R.D. Sharman, P.K. Smolarkiewicz, On physical realizability and uncertainty of numerical solutions, Atmos. Sci. Lett. (2005) 118-122.

[9] P.G. Drazin, W. H. Reid, Hydrodynamic stability, Cambridge University Press (1971) 527pp.

[10] P.D. Düben, P. Korn, Atmosphere and ocean modeling on grids of variable resolution - a 2D case study, Month. Weather Rev. 142 (2014) 1997-2017.

[11] C.C. Epifanio, R. Rotunno, The dynamics of orographic wake formation in flows with upstream blocking, J. Atmos. Sci. 62 (2005) 3127-3150.

[12] G. Fosser, S. Khodayar, P. Berg, Benefit of convection permitting climate model simulations in the representation of convective precipitation, Clim. Dyn. 44 (2015) 45-60.

[13] T. Gal-Chen, C.J. Somerville, On the use of a coordinate transformation for the solution of the Navier-Stokes equations, J. Comput. Phys. 17 (1975) 209-228. 
[14] M. Ghizaru, P. Charbonneau, P.K. Smolarkiewicz, Magnetic Cycles in Global Large-eddy Simulations of Solar Convection, Astrophys. J. Lett. 715 (2010) L133-L137

[15] T. Grätsch, K.J. Bathe, A posteriori error estimation techniques in practical finite element analysis, Comput. Struct. 83 (2005) 235-265.

[16] V. Grubisic, P.K. Smolarkiewicz, The Effect of Critical Levels on 3D Orographic Flows: Linear Regime, J. Atmos. Sci. 54 (1997) 1943-1969.

[17] D. Ham, M. Piggott, T. Ringler, H. Weller, N. Wood, Multiscale Numerics for the Atmospher and Ocean, Isaac Newton Institute for Mathematical Scinces, 22nd August-21st December 2012, https://www.newton.ac.uk/event/amm

[18] C.R. Hunt, W.H. Snyder, Experiments on stably and neutrally stratified flow over a model three-dimensional hill, J. Fluid Mech. 96 (1980) 671-704.

[19] J.D. Hyman, P.K. Smolarkiewicz, C.L. Winter, Heterogeneities of flow in stochastically generated porous media, Phys. Rev. E 86 (2012) 056701.

[20] J.B. Klemp, W.C. Skamarock, O. Fuhrer, Numerical consistency of metric terms in terrain-following coordinates, Month. Weather Rev. 131 (2003) 1229-1239.

[21] C. Kühnlein, P.K. Smolarkiewicz, A. Dörnbrack, Modelling atmospheric flows with adaptive moving meshes, J. Comput. Phys. 231 (2012) 2741-2763.

[22] F.B. Lipps, R.S. Hemler, A scale analysis of deep moist convection and some related numerical calculations, J. Atmos. Sci. 39 (1982) 2192-2210.

[23] F.B. Lipps, On the anelastic approximation for deep convection, J. Atmos. Sci. 47 (1990) 1794-1798.

[24] L.G. Margolin, W.J. Rider, A Rationale for Implicit Turbulence Modeling, Int. J. Num. Meth. Fluids 39 (2002) 821-841.

[25] L.G. Margolin, P.K. Smolarkiewicz, A.A. Wyszogrodzki, Implicit turbulence modeling for high Reynolds number flows. J. Fluids Eng. 124 (2002) 862-867.

[26] L.G. Margolin, W.J. Rider, and F.F. Grinstein, J. Turbul. 7 N15 (2006) 1-27.

[27] A.L. Marsden, O.V. Vasilyev, P. Moin, Construction of commutative filters for LES on unstructured meshes, J. Comput. Phys. 175 (2002) 584-603.

[28] S.A. Maslowe, Critical layers in shear flows. Annu. Rev. Fluid Mech. 18 (1986) 405-432.

[29] N. Nikiforakis, Mesh generation and mesh adaptation for large-scale Earthsystem modelling, Introduction, Phil. Trans. R. Soc. A 367 (2009) 4473-4481.

[30] E. Onate, J. Castro, Adaptive mesh refinement techniques for structural problems, The Finite Element Method in the 90's, eds. E. Onate et al, Springer, CIMNE, Barcelona (1990).

[31] J.M. Prusa, P.K. Smolarkiewicz, An all-scale anelastic model for geophysical flows: dynamic grid deformation, J. Comput. Phys. 190 (2003) 601-622. 
[32] J.M. Prusa, P.K. Smolarkiewicz, A.A Wyszogrodzki, EULAG, a computational model for multiscale flows, Comput. Fluids 37 (2008) 1193-1207

[33] W.J. Rider, The relationship of MPDATA to other high-resolution Methods. International Journal for Numerical Methods in Fluids 50 (2006), 11451158.

[34] C. Schär, D. Leuenberger, O. Fuhrer, D. Lüthi, C. Girard, A new terrainfollowing vertical coordinate formulation for atmospheric prediction models, Month. Weather Rev. 130 (2002) 2459-2480.

[35] R.B. Smith, Linear theory of stratified flow past an isolated mountain in isosteric coordinates, J. Atmos. Sci. 45 (1988) 3889-3896

[36] P.K. Smolarkiewicz, T.L. Clark, Numerical-simulation of the evolution of a 3dimensional field of cumulus clouds. Part I: Model description, comparison with observations and sensitivity studies, J. Atmos. Sci. 42 (1985) 502-521.

[37] P.K. Smolarkiewicz, R. Rotunno, Low Froude number flow past threedimensional obstacles. Part I: Baroclinically generated lee vortices, J. Atmos. Sci. 46 (1989) 1154-1164.

[38] P.K. Smolarkiewicz, V. Grubišić, L.G. Margolin, On forward-in-time differencing for fluids: stopping criteria for iterative solutions of anelastic pressure equations, Month. Weather Rev. 125 (1997) 647-654.

[39] P.K. Smolarkiewicz, L.G. Margolin, MPDATA: A finite-difference solver for geophysical flows, J. Comput. Phys. 140 (1998) 459-480.

[40] P.K. Smolarkiewicz, Multidimensional positive definite advection transport algorithm: an overview, Int. J. Numer. Meth. Fluids 50 (2006) 1123-1144.

[41] P.K. Smolarkiewicz, J. Szmelter, Multidimensional positive definite advection transport algorithm (MPDATA): an edge-based unstructured-data formulation, Int. J. Numer. Meth. Fluids 47 (2005) 1293-1299.

[42] P.K. Smolarkiewicz, J. Szmelter, MPDATA: An edge-based unstructured-grid formulation, J. Comput. Phys. 206 (2005) 624-649.

[43] P.K. Smolarkiewicz, J. Szmelter, Iterated upwind schemes for gas dynamics, J. Comput. Phys. 228 (2009) 33-54.

[44] P.K. Smolarkiewicz, J. Szmelter, A nonhydrostatic unstructured-mesh soundproof model for simulation of internal gravity waves, Acta Geophysica 59 (2011) 1109-1134.

[45] P.K. Smolarkiewicz, J. Szmelter, A.A. Wyszogrodzki, An unstructured-mesh atmospheric model for nonhydrostatic dynamics, J. Comput. Phys. 254 (2013) 184-199.

[46] P.K. Smolarkiewicz, C. Kühnlein, N.P. Wedi, A consistent framework for discrete integrations of soundproof and compressible PDEs of atmospheric dynamics, J. Comput. Phys. 263 (2014) 185-205 
[47] E.A. Spiegel, G. Veronis, On the Boussinesq approximation for compressible fluid, Astrophys. J. 131 (1960) 442-447.

[48] G.J. Steeneveld, R. Ronda, A.A.M. Holtslag, The challenge of forecasting the onset and development of radiation fog using mesoscale atmospheric models, Boundary-Layer Meteorol., 154 (2015) 265-289.

[49] J. Szmelter, M.J. Marchant, A. Evans, N.P. Weatherill, Two-dimensional Navier-Stokes equations with adaptivity on structured meshes,Comput. Method. Appl. M. 101 (1992) 355-368.

[50] J. Szmelter, P.K. Smolarkiewicz, MPDATA error estimator for mesh adaptivity, Int. J. Numer. Meth. Fluids 50 (2006) 1269-1293.

[51] J. Szmelter, P. Ortiz, Burning surfaces evolution in solid propellants: a numerical model, P. I. Mech. Eng. G-J. Aer. 221 (2007), 429-440.

[52] J. Szmelter, P.K. Smolarkiewicz, An edge-based unstructured mesh discretisation in geospherical framework, J. Comput. Phys. 229 (2010) 49804995 .

[53] J. Szmelter, P.K. Smolarkiewicz, An edge-based unstructured mesh framework for atmospheric flows, Comput. Fluids. 46 (2011) 455-460.

[54] E.F. Toro, Riemann Solvers and Numerical Methods for Fluid Dynamics, 3ed Edition, Springer, 2010, 724 pp.

[55] M.L. Waite, P.K. Smolarkiewicz, Instability and breakdown of vertical vortex pair in a strongly stratified fluid, J. Fluid. Mech. 606 (2008) 239-273.

[56] N.P. Wedi, P.K. Smolarkiewicz, Extending Gal-Chen and Somerville terrainfollowing coordinate transformation on time dependent curvilinear boundaries, J. Comput. Phys. 193 (2004) 1-20.

[57] N.P. Wedi, P.K. Smolarkiewicz, Direct numerical simulation of the PlumbMcEvan laboratory analog of the QBO, J. Atmos. Sci. 63 (2006) 3226-3252.

[58] D.L. Williamson, The evolution of dynamical cores for global atmospheric models, J. Meteor. Soc. Japan 85B (2007) 241-268.

[59] J. Wu, J.Z. Zhu, J. Szmelter, O.C. Zienkiewicz, Error Estimation and Adaptivity in Navier-Stokes Incompressible Flows, Comput. Mech. 6 (1990) 259-270.

[60] O.C. Zienkiewicz, B. Boroomand, J.Z. Zhu, Recovery procedures in error estimation and adaptivity: Adaptivity in linear problems, Advances in Adaptive Computational Methods in Mechanics, eds P. Ladeveze et al, Elsevier (1998). 